\title{
Optimized Power Control Design for Over-the-Air Federated Edge Learning
}

\author{
Xiaowen Cao, Student Member, IEEE, Guangxu Zhu, Member, IEEE, Jie Xu, Member, IEEE, Zhiqin Wang, and \\ Shuguang Cui, Fellow, IEEE
}

\begin{abstract}
Over-the-air federated edge learning (Air-FEEL) has emerged as a communication-efficient solution to enable distributed machine learning over edge devices by using their data locally to preserve the privacy. By exploiting the waveform superposition property of wireless channels, Air-FEEL allows the "one-shot" over-the-air aggregation of gradient-updates to enhance the communication efficiency, but at the cost of a compromised learning performance due to the aggregation errors caused by channel fading and noise. This paper investigates the transmission power control to combat against such aggregation errors in Air-FEEL. Different from conventional power control designs (e.g., to minimize the individual mean squared error (MSE) of the over-the-air aggregation at each round), we consider a new power control design aiming at directly maximizing the convergence speed. Towards this end, we first analyze the convergence behavior of Air-FEEL (in terms of the optimality gap) subject to aggregation errors at different communication rounds. It is revealed that if the aggregation estimates are unbiased, then the training algorithm would converge exactly to the optimal point with mild conditions; while if they are biased, then the algorithm would converge with an error floor determined by the accumulated estimate bias over communication rounds. Next, building upon the convergence results, we optimize the power control to directly minimize the derived optimality gaps under the cases without and with unbiased aggregation constraints, subject to a set of average and maximum power constraints at individual edge devices. We transform both problems into convex forms, and obtain their structured optimal solutions, both appearing in a form of regularized channel inversion, by using the Lagrangian duality method. Finally, numerical results show that the proposed power control policies achieve significantly faster convergence for Air-FEEL, as compared with benchmark policies with fixed power transmission or conventional MSE minimization.
\end{abstract}

Index Terms-Federated learning, over-the-air computation,

Part of this paper has been presented at the IEEE International Conference on Communications (ICC), Montreal, Canada, Jun. 14-23, 2021 [1]. This work was supported by the Key Area R\&D Program of Guangdong Province with grant No. 2018B030338001, the National Key R\&D Program of China with grant No. 2018YFB1800800, Shenzhen Outstanding Talents Training Fund, Guangdong Research Project No. 2017ZT07X152, the National Natural Science Foundation of China under grants U2001208, 61871137, and 62001310, and the Science and Technology Program of Guangdong Province under grant 2021A0505030002. Corresponding authors: Guangxu Zhu and Zhiqin Wang.

$\mathrm{X}$. Cao is with the School of Information Engineering, Guangdong University of Technology, Guangzhou, China, and the Future Network of Intelligence Institute (FNii), The Chinese University of Hong Kong (Shenzhen), Shenzhen, China (e-mail: caoxwen@outlook.com).

G. Zhu is with Shenzhen Research Institute of Big Data, Shenzhen, China (e-mail: gxzhu@sribd.cn).

J. Xu is with the School of Science and Engineering (SSE) and the FNii, The Chinese University of Hong Kong (Shenzhen), Shenzhen, China (e-mail: xujie@cuhk.edu.cn).

Z. Wang is with China Academy of Information and Communications Technology, Beijing, China (e-mail: zhiqin.wang@ caict.ac.cn).

S. Cui is with the SSE and the FNii, The Chinese University of Hong Kong (Shenzhen), Shenzhen, China, and Shenzhen Research Institute of Big Data, Shenzhen, China. (e-mail: shuguangcui@cuhk.edu.cn). stochastic gradient descent, power control, edge intelligence.

\section{INTRODUCTION}

In the pursuit of ubiquitous brain-inspired intelligence envisioned in the future $6 \mathrm{G}$ networks [2], recent years have witnessed the spreading of artificial intelligence (AI) algorithms from the cloud to the network edge, resulting in an active area called edge intelligence [3], [4]. The core research issue therein is to allow low-latency and privacy-aware access to rich mobile data for intelligence distillation. To this end, the federated edge learning (FEEL) framework has been proposed recently, which distributes the AI-model training task over edge devices by using their data locally to preserve the privacy [5]-[10]. Essentially, the FEEL framework corresponds to the implementation of distributed gradient descent over wireless networks. Such a training process is to find optimized AImodel parameters by minimizing a properly designed loss function in an iterative manner. Specifically, at each iteration or communication round, the edge server first broadcasts the global AI-model parameters to edge devices, such that all edge devices can synchronize their local models; next, the edge devices compute their respective local gradient updates using the local data and then upload them to the edge server for further aggregation to update the global model. Although the uploading of high-volume raw data is avoided, the gradient aggregation process in FEEL may still suffer from a communication bottleneck due to the high dimensionality of each gradient update, especially when the number of edge devices sharing the same wireless medium becomes large. To tackle this issue, one promising solution called over-the-air FEEL (Air-FEEL) has been proposed (see, e.g., [11]), which exploits the over-the-air computation (AirComp) technique for "oneshot" aggregation by allowing multiple devices' concurrent update transmission. In such a way, the communication and computation are integrated in a joint design by exploiting the superposition property of a multiple access channel (MAC) [12], [13].

The idea of AirComp was first proposed in [12] for data aggregation in sensor networks, which harnessed the "interference" via structured codes to help functional computation over a MAC. The subsequent work [13] showed that for Gaussian independent and identically distributed (i.i.d.) data sources, the uncoded (analog) transmission is optimal to minimize the distortion in AirComp. Building on the information-theoretic studies, the analog AirComp implementation has attracted growing research interests (see, e.g., [14]-[21]). For instance, the synchronization issue in AirComp was addressed in [14] 
via a shared clock broadcasting from edge server to devices; the optimal power control policies for AirComp over fading channels were derived in [15], [16] to minimize the average computation distortion; and the cooperative interference management for multi-cell AirComp networks was developed in [17]. Furthermore, multiple-input-multiple-output (MIMO) spatial multiplexing was exploited in AirComp to enable vector-valued functional computation targeting multi-modal sensing [18], [19] and to enhance the computational accuracy [20], [21].

Recently, AirComp found its merits in the new context of FEEL, namely the Air-FEEL, to enable the communicationefficient gradient aggregation at each communication round [22]. Prior works studied the Air-FEEL system from different perspectives such as devices scheduling [23]-[26], beamforming design [27], [28], update compression [29]-[31], and hyper-parameters (such as learning rates) optimization [32]-[34]. For instance, a broadband Air-FEEL solution was proposed in [23], where a set of communication-learning tradeoffs were derived to guide the device scheduling. Along this vein, the authors in [24] proposed an energy-aware device scheduling strategy to minimize the expected improvement of loss function value at each communication round, while the authors in [25] proposed a threshold-based device selection scheme to achieve reliable model aggregation. Then, for multi-antenna Air-FEEL systems, a joint design of device scheduling and receive beamforming was presented in [27], and a unit-modulus analog receive beamforming design was proposed in [28]. As for update compression, a source-coding algorithm exploiting gradient sparsification was proposed in [29], [30], and a compressive-sensing based gradient aggregation approach was developed in [31] to further improve the communication efficiency. Lately, Air-FEEL based on digital modulation was proposed in [35] and further extended in [36], which features one-bit quantization and modulation at the edge devices and majority-vote based decoding at the edge server. Besides improving the communication efficiency, the Air-FEEL has also been exploited for enhancing the data privacy, in which individual updates are not accessible by the centralized edge server, thus eliminating the risk of potential model inversion attack [37]-[40].

Generally speaking, the employment of AirComp introduces an essential design tradeoff in Air-FEEL between the the enhanced communication efficiency (via the over-the-air aggregation) and the degraded learning performance (due to the aggregation error caused by the channel fading and noise perturbation). Due to such a tradeoff, how to analytically characterize the training performance (e.g., in terms of accuracy and latency) is a challenging task has not been investigated in the literature yet. Also notice that the aggregation distortion in different communication rounds may have distinct impacts on the learning performance [41], thus making the above tradeoff even more complicated. To deal with these issues, it is crucial to properly control the transmission power at different edge devices over different communication rounds. In the literature, there have been prior works on Air-FEEL [23]-[27], [29], [33], [37] that considered simplified channel inversion (or its variants) to align the channel gains among different devices, which, however, may lead to amplified noise at receiver and thus is highly suboptimal especially in deep fading scenarios. Some other works [34], [42] designed the power control with the objective of minimizing the individual aggregation distortion (e.g., mean squared error (MSE)) at each communication round, which, however, may not perform well as the aggregation distortion in different communication rounds may have distinct impact on the learning performance [41]. Therefore, how to obtain the analytic learning performance of Air-FEEL in terms of the power control variables, and accordingly optimize the power control decisions for optimizing the learning performance still remains unknown. This thus motivates the current work.

This paper studies an Air-FEEL system consisting of multiple edge devices and one edge server. By considering smooth learning models satisfying the Polyak-Łojasiewicz inequality, we establish an elegant learning performance metric, namely the optimality gap, linking with the aggregation errors over communication rounds. Accordingly, we propose optimized power control policies for directly minimizing the optimality gap. The main contributions are summarized as follows.

- Convergence analysis: First, we analyze the optimality gap of the loss function over different communication rounds, which characterizes the impact of gradient aggregation errors (i.e., the bias and MSE of the gradient aggregation estimates) on the convergence performance of the Air-FEEL algorithm. It is revealed that if the aggregation errors are unbiased, the optimality gap will diminish to zero with sufficiently many communication rounds and properly chosen step sizes; while if the aggregation errors are biased, the optimality gap would reach to an error floor whose height is equal to the accumulated estimate bias over communication rounds. It is also shown that within a finite number of communication rounds, the aggregation errors at later rounds (with higher weights) contribute more to the optimality gap than those at earlier rounds.

- Power control optimization: Next, building on the convergence results, we formulate new power control optimization problems for Air-FEEL under the cases without and with unbiased aggregation constraints, respectively, with the objective of minimizing the optimality gap, subject to a set of average and maximum power constraints at individual edge devices. Fortunately, both power control problems can be transformed into convex forms, which can thus be optimally solved by the Lagrangian duality method. The optimized power control solutions establish a regularized channel inversion structure, where the regularization term at each edge device is related to all other devices' average power budgets for the case without unbiased aggregation constraints, and is only related to the own device's individual average power budget for the case with unbiased aggregation constraints.

- Performance evaluation: Finally, we conduct extensive simulations to evaluate the performance of the optimized power control for Air-FEEL by considering the ridge regression with synthetic dataset, and handwritten digit recognition using MNIST dataset with a convolution 
neural network $(\mathrm{CNN})$. It is shown that the proposed power control policies in the cases without and with unbiased aggregation constraints achieve significantly faster convergence rate (or lower optimality gap), than the benchmarking fixed power transmission and conventional MSE minimization schemes, as the proposed policies can better handle the aggregation errors over rounds based on their contributions to the optimality gap. It is also shown that the the case without unbiased aggregation constraints can achieve lower optimality gap than that with biased aggregation constaints when some mild conditions are met. This validates our analysis that the Air-FEEL can always converge to the optimal point with unbiased gradient aggregation.

Notations: Bold lowercase letters refer to column vectors. $\mathbb{E}(\cdot)$ denotes the expectation operation; the superscript $T$ represents the transpose operation; $\nabla$ is the gradient operator, and $(x)^{+} \triangleq \max \{0, x\}$. For a set $\mathcal{A},|\mathcal{A}|$ denotes its cardinality. $\|\boldsymbol{a}\|$ denotes the Euclidean norm of vector $\boldsymbol{a}$. I denotes the identity matrix. For ease of reference, the main notations used in this paper are listed in Table [.

Table I

List OF MAIN NOTATIONS

\begin{tabular}{|c|l|}
\hline Symbol & Description \\
\hline $\mathcal{K}$ & Set of edge devices with $\mathcal{K} \triangleq\{1, \ldots, K\}$ \\
\hline $\mathcal{N}$ & Set of communication rounds with $\mathcal{N} \triangleq\{1, \cdots, N\}$ \\
\hline $\mathbf{w} \in \mathbb{R}^{q}$ & Parameter vector of learning model with size $q$ \\
\hline $\mathbf{w}^{\star}$ & Optimal parameter vector \\
\hline $\mathcal{D}_{k}$ & Local dataset at edge device $k \in \mathcal{K}$ with cardinality $D_{k}$ \\
\hline$\left(\mathbf{x}_{i}, \tau_{i}\right)$ & The $i$-th sample $\mathbf{x}_{i}$ in dataset with ground-true label $\tau_{i}$ \\
\hline$f\left(\mathbf{w}, \mathbf{x}_{i}, \tau_{i}\right)$ & $\begin{array}{l}\text { Sample-wise loss function for quantifying the prediction } \\
\text { error of the learning model } \mathbf{w} \text { on } \mathbf{x}_{i} \text { in terms of } \tau_{i}\end{array}$ \\
\hline$F_{k}(\mathbf{w})$ & $\begin{array}{l}\text { Local loss function of the learning model vector } \mathbf{w} \text { on } \mathcal{D}_{k} \\
\text { at edge device } k \in \mathcal{K}\end{array}$ \\
\hline$F(\mathbf{w})$ & Global loss function at the parameter model $\mathbf{w}$ \\
\hline $\mathbf{g}_{k}^{(n)}$ & $\begin{array}{l}\text { Local gradient estimate in edge device } k \text { at communica- } \\
\text { tion round } n\end{array}$ \\
\hline$\overline{\mathbf{g}}^{(n)}$ & Global gradient estimation at communication round $n$ \\
\hline$\hat{\mathbf{g}}^{(n)}$ & $\begin{array}{l}\text { Global gradient received at the edge server through over- } \\
\text { the-air aggregation at communication round } n\end{array}$ \\
\hline$\eta^{(n)}$ & Learning rate at communication round $n$ \\
\hline$\varepsilon^{(n)}$ & Aggregation error at each communication round $n$ \\
\hline$\nabla F(\mathbf{w})$ & $\begin{array}{l}\text { Ground-truth gradient of the loss function evaluated at } \\
\text { point } \mathbf{w} \in \mathbb{R}^{q}\end{array}$ \\
\hline$F^{\star}$ & Optimal loss function value that is equal to $F\left(\mathbf{w}{ }^{\star}\right)$ \\
\hline$\hat{h}_{k}^{(n)}$ & $\begin{array}{l}\text { Complex channel coefficient from edge device } k \in \mathcal{K} \text { to } \\
\text { the edge server at communication round } n\end{array}$ \\
\hline $\mathbf{z}^{(n)} \in \mathbb{R}^{q}$ & AWGN with $\mathbf{z}^{(n)} \sim \mathcal{C} N\left(0, \sigma_{z}^{2} \mathbf{I}\right)$ \\
\hline$p_{k}^{(n)}$ & $\begin{array}{l}\text { Power scaling factor in edge device } k \in \mathcal{K} \text { at communi- } \\
\text { cation round } n\end{array}$ \\
\hline $\mathbf{y}^{(n)}$ & Received signal in edge server at communication round $n$ \\
\hline
\end{tabular}

\section{SySTEM MODEL}

We consider an Air-FEEL system consisting of an edge server and $K$ edge devices, as shown in Fig. 1 . With the coordination of the edge server, the edge devices cooperatively train a shared machine learning model via the over-the-air gradient aggregation as elaborated in the sequel.

\section{A. Learning Model}

We assume that the learning model is represented by the parameter vector $\mathbf{w} \in \mathbb{R}^{q}$ with $\mathbf{w}=\left[w_{1}, \cdots, w_{q}\right]^{T}$ and $q$

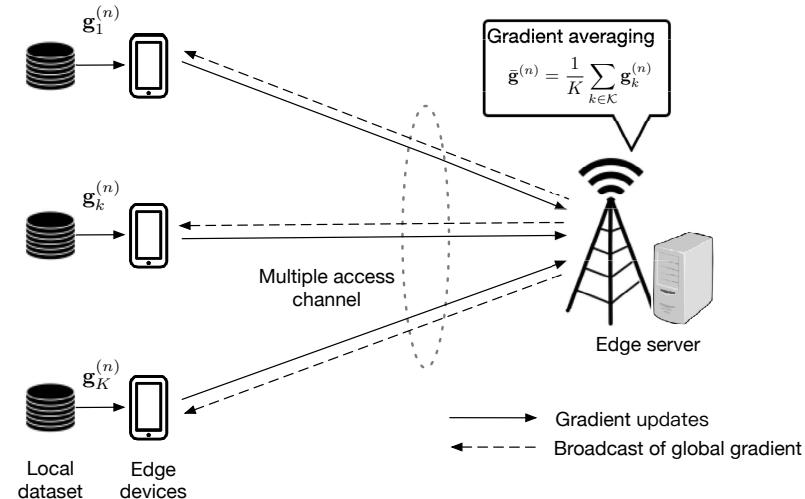

Figure 1. Illustration of over-the-air federated edge learning (Air-FEEL).

denoting the learning model size. Let $\mathcal{D}_{k}$ denote the local dataset at edge device $k$, in which the $i$-th sample and its ground-true label are denoted by $\mathbf{x}_{i}$ and $\tau_{i}$, respectively. Define $f\left(\mathbf{w}, \mathbf{x}_{i}, \tau_{i}\right)$ as the sample-wise loss function quantifying the prediction error of the learning model $\mathbf{w}$ on sample $\mathbf{x}_{i}$ with respect to (w.r.t.) its ground-true label $\tau_{i}$. Then the local loss function of the learning model vector $\mathbf{w}$ on $\mathcal{D}_{k}$ is

$$
F_{k}(\mathbf{w})=\frac{1}{\left|\mathcal{D}_{k}\right|} \sum_{\left(\mathbf{x}_{i}, \tau_{i}\right) \in \mathcal{D}_{k}} f\left(\mathbf{w}, \mathbf{x}_{i}, \tau_{i}\right)
$$

For notational convenience, we denote $f\left(\mathbf{w}, \mathbf{x}_{i}, \tau_{i}\right)$ as $f_{i}(\mathbf{w})$ and assume that the sizes of local datasets at different edge devices are uniform, i.e., $D \triangleq D_{k}=\left|\mathcal{D}_{k}\right|, \forall k \in \mathcal{K}$. Then, the global loss function on all the distributed datasets $\mathcal{D}_{\text {tot }}=$ $\cup_{k \in \mathcal{K}} \mathcal{D}_{k}$ evaluated on parameter vector $\mathbf{w}$ is given by

$$
F(\mathbf{w})=\frac{1}{D_{\text {tot }}} \sum_{k \in \mathcal{K}} D_{k} F_{k}(\mathbf{w})=\frac{1}{K} \sum_{k \in \mathcal{K}} F_{k}(\mathbf{w}),
$$

where $D_{\text {tot }}=\left|\mathcal{D}_{\text {tot }}\right|=K D$.

The objective of the training process is to find a desired parameter vector $\mathbf{w}$ for minimizing the global loss function $F(\mathbf{w})$ in (2), i.e.,

$$
\mathbf{w}^{\star}=\arg \min _{\mathbf{w}} F(\mathbf{w}) \text {. }
$$

Instead of uploading all the local data to the edge server for centralized training, we consider the Air-FEEL, in which the learning process in (3) is implemented iteratively in a distributed manner using the federated stochastic gradient descent (FedSGD) algorithm [1 [44] as detailed in the following.

Consider a particular iteration or communication round $n$, with the learning model before updating being denoted by $\mathbf{w}^{(n)}$. In this round, each edge device $k \in \mathcal{K}$ computes the local gradient estimate of the loss function as $\mathbf{g}_{k}^{(n)}$, based on a randomly sampled mini-batch from the local dataset. We denote the set of mini-batch data used by the edge device $k$

\footnotetext{
${ }^{1}$ Besides the FedSGD, the federated averaging (FedAvg) is an alternative method for Air-FEEL, which can be implemented with multiple local updates at edge devices together with the model averaging at the edge server. This paper considers the FedSGD (instead of FedAvg), mainly for the purpose of facilitating the convergence analysis and gaining insightful results. Furthermore, the FedSGD enjoys the advantages of being more robust to the non-i.i.d. data [43]. Nevertheless, our design and analysis principles in this paper can be extended to the case with FedAvg, which is left for future work.
} 
at round $n$ as $\tilde{\mathcal{D}}_{k}^{(n)}$ and its size $m_{b}=\left|\tilde{\mathcal{D}}_{k}^{(n)}\right|, \forall k \in \mathcal{K}$. Then we have

$$
\mathbf{g}_{k}^{(n)}=\frac{1}{m_{b}} \sum_{\left(\mathbf{x}_{i}, \tau_{i}\right) \in \tilde{\mathcal{D}}_{k}} \nabla f_{i}\left(\mathbf{w}^{(n)}\right) .
$$

Next, the edge devices upload their local gradients to the edge server for aggregation. If the aggregation is error-free, then the global gradient estimate can be obtained as an average of local gradient estimates from all different edge devices, i.e.2

$$
\overline{\mathbf{g}}^{(n)}=\frac{1}{K} \sum_{k \in \mathcal{K}} \mathbf{g}_{k}^{(n)}
$$

Then, the edge server broadcasts the obtained global gradient estimate $\overline{\mathbf{g}}^{(n)}$ to the edge devices, based on which different edge devices can synchronously update their own learning model via

$$
\mathbf{w}^{(n+1)}=\mathbf{w}^{(n)}-\eta^{(n)} \cdot \overline{\mathbf{g}}^{(n)}
$$

where $\eta^{(n)}$ is the learning rate at communication round $n$. The above procedure continues until the convergence criteria is met or the maximum number of communication rounds is achieved.

Notice that this paper considers the over-the-air aggregation approach to achieve fast gradient aggregation, based on which the received aggregated gradient at the edge server in (5) may be erroneous due to perturbation caused by the channel fading and noise. This issue will be elaborated in Section II-C.

\section{B. Basic Assumptions on Learning Model}

To facilitate the convergence analysis, we make several assumptions on the loss functions and gradient estimates, which are commonly made in the literature [25], [28], [34], [37], [45], [46].

Assumption 1 (Smoothness). Let $\nabla F(\mathbf{w})$ denote the gradient of the loss function evaluated at point $\mathbf{w} \in \mathbb{R}^{q}$. Then there exists a non-negative constant vector $\mathbf{L} \in \mathbb{R}^{q}$ with $\mathbf{L}=\left[L_{1}, \cdots, L_{q}\right]^{T}$, such that

$$
\begin{aligned}
& F(\mathbf{w})-\left[F\left(\mathbf{w}^{\prime}\right)+\nabla F(\mathbf{w})^{T}\left(\mathbf{w}-\mathbf{w}^{\prime}\right)\right] \\
& \leq \frac{1}{2} \sum_{i=1}^{q} L_{i}\left(w_{i}-w_{i}^{\prime}\right)^{2}, \forall \mathbf{w}, \mathbf{w}^{\prime} \in \mathbb{R}^{q} .
\end{aligned}
$$

Assumption 1 guarantees that the gradient of the loss function would not change arbitrarily quickly w.r.t. the parameter vector. Note that such an assumption is essential for convergence analysis of gradient decent methods to provide a good indicator for how far to decrease to the minimum loss.

Assumption 2 (Polyak-Łojasiewicz inequality). Let $F^{\star}$ denote the optimal loss function value to problem (3). There exists a constant $\delta \geq 0$ such that the global loss function $F(\mathbf{w})$ satisfies the following Polyak-Łojasiewicz condition:

$$
\|\nabla F(\mathbf{w})\|^{2} \geq 2 \delta\left(F(\mathbf{w})-F^{\star}\right) .
$$

\footnotetext{
${ }^{2}$ Although we consider the same data size $D$ at different edge devices, our proposed Air-FEEL can be easily extended to the case when they have different data sizes, i.e., $D_{k}$ 's are different. In this case, we only need to revise the global gradient estimate in (5) as a weighted-average of the local ones, i.e., $\overline{\mathbf{g}}^{(n)}=\sum_{k \in \mathcal{K}} \frac{D_{k}}{D_{\text {tot }}} \mathbf{g}_{k}^{(n)}$. Via AirComp, the desired weighted aggregation of the local gradient estimate can be easily attained by adding an additional pre-processing $\psi(\cdot)$ on the transmitted signal $s_{k}$ with $\psi\left(s_{k}\right)=\sum_{k \in \mathcal{K}} \frac{D_{k}}{D_{\text {tot }}} s_{k}$.
}

Notice that Assumption 2 is more general than the standard assumption of strong convexity [47]. The inequality in (7) simply requires that the gradient grows faster than a quadratic function when away from the optimal function value and implies that every stationary point is a global minimum. Typical loss functions satisfying Assumptions 1 and 2 include logistic regression, linear regression, and least squares.

Assumption 3 (Variance bound). The local gradient estimates $\left\{\mathbf{g}_{k}\right\}$, defined in (4), where the index $n$ is omitted for simplicity, are assumed to be independent and unbiased estimates of the batch gradient $\nabla F(\mathbf{w})$ with coordinate bounded variance, i.e.,

$$
\begin{aligned}
& \mathbb{E}\left[\mathbf{g}_{k}\right]=\nabla F(\mathbf{w}), \forall k \in \mathcal{K}, \\
& \mathbb{E}\left[\left(g_{k, i}-\nabla F\left(w_{i}\right)\right)^{2}\right] \leq \frac{\sigma_{i}^{2}}{m_{b}}, \forall k \in \mathcal{K}, \forall i,
\end{aligned}
$$

where $g_{k, i}$ and $\nabla F\left(w_{i}\right)$ are defined as the $i$-th element of $\left\{\mathbf{g}_{k}\right\}$ and $\nabla F(\mathbf{w})$, respectively, $\boldsymbol{\sigma}=\left[\sigma_{1}, \cdots, \sigma_{q}\right]$ is a vector of non-negative constants, and the denominator $m_{b}$ accounts for the fact that the local gradient estimate is computed over a mini-batch of data with size $m_{b}$.

Notice that the following convergence analysis and power control optimization in Sections [II] and [V] are based on Assumptions 1] 3, similarly as in prior work [10], [25], [28], [34], [37]. Nevertheless, as shown in simulations in Section V-C] the proposed power control designs can still work well for CNN when such assumptions are relaxed.

\section{Over-the-Air Aggregation for FEEL}

The distributed training latency for FEEL is dominated by the update aggregation process, especially when the number of devices becomes large. Therefore, we focus on the aggregation process over a MAC. To accelerate the learning, we employ the AirComp technique for fast gradient aggregation by exploiting the superposition property of the MAC. To implement AirComp, during the gradient-uploading phase, all devices transmit simultaneously over the same time-frequency block with proper phase compensation. For ease of exposition, it is assumed that the channel coefficients remain unchanged within each communication round, but may change over different rounds. It is also assumed that the edge devices can perfectly know their own channel state information (CSI), so they can compensate for the channel phase differences.

Let $\hat{h}_{k}^{(n)}$ denote the complex channel coefficient from edge device $k$ to the edge server at communication round $n$, and $h_{k}^{(n)}$ denote its post-compensated real-valued channel coefficient, i.e., $h_{k}^{(n)}=\left|\hat{h}_{k}^{(n)}\right|$. Then, the received aggregated signal via AirComp (after phase compensation) is given by

$$
\mathbf{y}^{(n)}=\sum_{k \in \mathcal{K}} h_{k}^{(n)} \sqrt{p_{k}^{(n)}} \mathbf{g}_{k}^{(n)}+\mathbf{z}^{(n)}
$$

in which $p_{k}^{(n)}$ denotes the power scaling factor at edge device $k$, and $\mathbf{z}^{(n)} \in \mathbb{R}^{q}$ denotes the additive white Gaussian noise (AWGN) with $\mathbf{z}^{(n)} \sim \mathcal{C N}\left(0, \sigma_{z}^{2} \mathbf{I}\right)$ and $\sigma_{z}^{2}$ being the noise power. Based on (10), the global gradient estimate at the edge 
server is given by

$$
\hat{\mathbf{g}}^{(n)}=\frac{\mathbf{y}^{(n)}}{K} .
$$

It thus follows from (10) and (11) that the aggregation error caused by the over-the-air aggregation in global gradient estimation is given by

$$
\begin{aligned}
\varepsilon^{(n)} & =\underbrace{\hat{\mathbf{g}}^{(n)}-\overline{\mathbf{g}}^{(n)}}_{\text {Signal misalignment error }} \\
& =\underbrace{\frac{1}{K} \sum_{k \in \mathcal{K}}\left(h_{k}^{(n)} \sqrt{p_{k}^{(n)}}-1\right) \mathbf{g}_{k}^{(n)}}_{\text {Noise }}+\underbrace{\frac{\mathbf{z}^{(n)}}{K}},
\end{aligned}
$$

which consists of two components representing the signal misalignment error and noise-induced error, respectively.

The devices can adaptively adjust their transmit powers by controlling $\left\{p_{k}^{(n)}\right\}$ to reduce the aggregation errors for enhancing the learning performance. We consider that each edge device $k \in \mathcal{K}$ is subject to a maximum power budget $\hat{P}_{k}^{\max }$ for each communication round, and an average power budget denoted by $\hat{P}_{k}^{\text {ave }}$ over the whole training period. Therefore, we have

$$
\frac{1}{q} \mathbb{E}\left(\left\|\sqrt{p_{k}^{(n)}} \mathbf{g}_{k}^{(n)}\right\|^{2}\right) \leq \hat{P}_{k}^{\max }, \forall k \in \mathcal{K}, \forall n \in \mathcal{N},
$$

where $q$ is the size of the gradient vector $g_{k}^{(n)}$, as well as

$$
\frac{1}{N q} \sum_{n \in \mathcal{N}} \mathbb{E}\left(\left\|\sqrt{p_{k}^{(n)}} \mathbf{g}_{k}^{(n)}\right\|^{2}\right) \leq \hat{P}_{k}^{\text {ave }}, \forall k \in \mathcal{K},
$$

where $\mathcal{N} \triangleq\{1, \cdots, N\}$ with $N$ denoting the total number of communication rounds for model training.

In the following Section III, we will establish a direct learning performance metric, namely the optimality gap, linking with the aggregation errors over communication rounds. Based on the analysis, in Section IV we will propose to minimize the optimality gap via optimizing the power control subject to a set of individual maximum and average power constraints.

\section{CONVERgence AnAlysis}

In this section, we present a convergence analysis framework for the FEEL in the presence of aggregation errors by using the optimality gap as the performance metric, which sheds light on how the imperfect gradient updates affect the convergence of FEEL in general. As will be shown shortly, depending on whether the aggregated gradient estimate is unbiased or not, the FEEL will have different convergence behaviors.

\section{A. Optimality Gap versus Aggregation Errors}

Suppose that at each communication round $n, F\left(\mathbf{w}^{(n)}\right)$ is the value of loss function w.r.t. the parameter vector $\mathbf{w}^{(n)}$. Thus, with the lossy gradient aggregation in (12), the update of learning model at communication round $n$ in (6) is represented as

$$
\mathbf{w}^{(n+1)}=\mathbf{w}^{(n)}-\eta^{(n)} \cdot\left(\overline{\mathbf{g}}^{(n)}+\varepsilon^{(n)}\right),
$$

\footnotetext{
${ }^{3}$ Unlike the conventional AirComp, using an additional scaling factor at the receiver, in 11 we directly use $\mathbf{y}^{(n)} / K$ as the estimated value of global gradient for Air-FEEL. This is due to the fact that the learning rate $\eta^{(n)}$ in 6. can play the equivalent role of scaling factor, and thus dedicated scaling factors are not needed as in conventional AirComp.
}

where $\varepsilon^{(n)}$ represents the induced random aggregation error (including the signal misalignment error and noice-induced error) at each communication round $n$. Let $\mathbb{E}\left[\varepsilon^{(n)}\right]$ and $\mathbb{E}\left[\left\|\varepsilon^{(n)}\right\|^{2}\right]$ denote the bias and MSE of the global gradient estimate at each communication round $n$, respectively, where the expectation operation is taken over the stochastic sample selection on the local gradient estimation over a mini-batch dataset, as well as the receiver noise due to AirComp.

Depending on the value of $\mathbb{E}\left[\varepsilon^{(n)}\right]$, we define two cases for the gradient aggregation.

- Case I without unbiased aggregation constraints: The aggregation can either be biased (i.e., $\mathbb{E}\left[\varepsilon^{(n)}\right] \neq 0$ ) or unbiased $\left(\mathbb{E}\left[\varepsilon^{(n)}\right]=0\right)$. In this case, no additional constraints on the aggregation biasness are introduced during the power control designs.

- Case II with unbiased aggregation constraints: The aggregation is unbiased, i.e., the constraints $\mathbb{E}\left[\varepsilon^{(n)}\right]=$ $0, \forall n \in \mathcal{N}$, are introduced in the aggregation designs (e.g., transmission power control).

Define the optimality gap after $N$ communication rounds as $F\left(\mathbf{w}^{(N+1)}\right)-F^{\star}$ and $L \triangleq\|\mathbf{L}\|_{\infty}$. Then, by considering a properly chosen fixed learning rate, we establish the following theorem.

Theorem 1 (Impact of aggregation error on convergence with a fixed learning rate). Under Assumption 1, suppose that the FEEL algorithm is implemented with a fixed learning rate $\eta \triangleq$ $\eta^{(n)}, \forall n \in \mathcal{N}$, with $0 \leq \eta \leq \frac{2}{2+L} \leq \frac{1}{\delta}$ and fixed mini-batch size $m_{b}=N$ [48]. Then, the expected optimality gap satisfies the inequality (16), where $C=1-\delta \eta$ with $0<C<1$.

\section{Proof: See Appendix A}

Remark 1. From Theorem 11, we have the following observations.

- The FEEL algorithm converges eventually as $N \rightarrow \infty$, with the optimality gap possibly landed on an error floor instead of diminishing to zero. It is observed from (16) that the upper bound of the optimality gap can be decomposed into two components, i.e., the error floor $\sum_{n \in \mathcal{N}} \frac{C^{N-n}}{2}\left\|\mathbb{E}\left[\varepsilon^{(n)}\right]\right\|^{2}$ that cannot vanish as $N$ grows, and the gap to the error floor, denoted by $\Delta(N)$, which can approach zero as $N$ increases. To see this, $\Delta(N)$ is observed to contain four terms related to the initial optimality gap $\left(\mathbb{E}\left[F\left(\mathbf{w}^{(1)}\right)\right]-F^{\star}\right)$, the gradient variance $\frac{\eta^{2} L\|\boldsymbol{\sigma}\|_{2}^{2}}{2 \delta N K^{2}}$, as well as the bias $\mathbb{E}\left[\varepsilon^{(n)}\right]$ and MSE $\mathbb{E}\left[\left\|\varepsilon^{(n)}\right\|^{2}\right]$ of the aggregation errors, respectively. All the four terms diminish as $N$ goes to infinity, or become negligible under a sufficiently small learning rate. On the other hand, the error floor is determined by the accumulated bias $\left\|\mathbb{E}\left[\varepsilon^{(n)}\right]\right\|^{2}$ over rounds. Hence, as $N$ increases, the error floor would approach a constant while the gap to it $\Delta(N)$ would vanish. Such effects are illustrated in Fig. 2

- The FEEL algorithm shows different convergence behaviors depending on whether the gradient aggregation is biased or not. For the case with unbiased aggregation constraints, i.e., $\mathbb{E}\left[\varepsilon^{(n)}\right]=0, \forall n \in \mathcal{N}$, as 


$$
\begin{aligned}
& \mathbb{E}\left[F\left(\mathbf{w}^{(N+1)}\right)\right]-F^{\star} \leq \underbrace{\sum_{n \in \mathcal{N}} \frac{C^{N-n}}{2}\|\underbrace{\mathbb{E}\left[\boldsymbol{\varepsilon}^{(n)}\right]}_{\text {Bias }}\|^{2}}_{\text {Error floor }}+ \\
& \underbrace{C^{N} \underbrace{\left(\mathbb{E}\left[F\left(\mathbf{w}^{(1)}\right)\right]-F^{\star}\right)}_{\text {The gap to the error floor } \Delta(N)}+\sum_{n \in \mathcal{N}}^{\sum_{\text {Gradient variance }} \frac{C^{N-n}}{2}}(\underbrace{\frac{\eta^{2} L\|\boldsymbol{\sigma}\|_{2}^{2}}{2 \delta N K^{2}}}_{\text {Bias }}+\eta^{2} L^{2} \leq \underbrace{\mathbb{E}\left[\varepsilon^{(n)}\right]}_{\text {MSE }} \|^{2}+\underbrace{2} L \underbrace{\mathbb{E}\left[\left\|\varepsilon^{(n)}\right\|^{2}\right]}}_{\text {Initial optimality gap }} .
\end{aligned}
$$

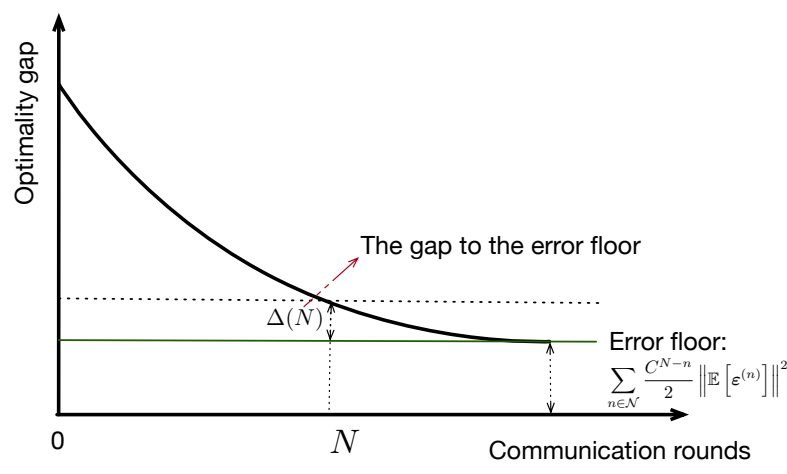

Figure 2. Illustration of contraction region on the learning performance.

$N$ becomes sufficiently large, the model under training can converge exactly to the optimal point with minimum training loss with zero error floor in the training process. By contrast, for the case without unbiased aggregation constraints, the model under training may only converge to a neighborhood of the optimal point (if the aggregation is biased). However, the case with unbiased aggregation constraints may converge slower compared with its counterpart without unbiased aggregation constraints, as the enforcement of the unbiasness generally comes at a cost of elevated MSE that translates to a larger gap to the error floor $\Delta(N)$. The observation is also validated via experiments shown in Section $\nabla$

- Latter rounds are more sensitive to aggregation error. The bias $\mathbb{E}\left[\varepsilon^{(n)}\right]$ and MSE $\mathbb{E}\left[\left\|\varepsilon^{(n)}\right\|^{2}\right]$ at the later communication rounds (with large $n$ ) contribute more on the optimality gap than that of the initial rounds (with small $n$ ), as the effect of the aggregation error introduced at early stages ( small $n$ ) is discounted by $C^{N-n}$ on the right hand side of (16).

Theorem 1 can be extended to the case with diminishing learning rates, as shown in the following corollary.

Corollary 1 (Impact of aggregation error on convergence with diminishing learning rates). Under Assumption 11 suppose that the FEEL algorithm is implemented with fixed minibatch size $m_{b}=N$, and diminishing learning rates $\eta^{(n)}=$ $\frac{u}{n+v}, \forall n \in \mathcal{N}$ [49], with $v>0$ and $u>1 / \delta$, such that $\eta^{(1)} \leq \frac{2}{2+L}$. Then, the expected optimality gap satisfies the following inequality:

$$
\begin{aligned}
& \mathbb{E}\left[F\left(\mathbf{w}^{(N+1)}\right)\right]-F^{\star} \leq\left(\prod_{n \in \mathcal{N}} C^{(n)}\right)\left(\mathbb{E}\left[F\left(\mathbf{w}^{(1)}\right)\right]-F^{\star}\right) \\
& +\sum_{n=1}^{N} J^{(n)}\left(\frac{\left(\eta^{(n)}\right)^{2} L\|\boldsymbol{\sigma}\|_{2}^{2}}{2 m_{b} K^{2}}+\left(\eta^{(n)}\right)^{2} L^{2}\left\|\mathbb{E}\left[\varepsilon^{(n)}\right]\right\|^{2}\right) \\
& +\sum_{n=1}^{N} J^{(n)}\left\|\mathbb{E}\left[\varepsilon^{(n)}\right]\right\|^{2}+\sum_{n=1}^{N} J^{(n)}\left(\eta^{(n)}\right)^{2} L \mathbb{E}\left[\left\|\varepsilon^{(n)}\right\|^{2}\right],(17) \\
& \text { where } C^{(n)}=1-\delta \eta^{(n)} \text { and } J^{(n)} \triangleq \frac{\prod_{i=n}^{N} C^{(i)}}{2 C^{(n)}}, \forall n \in \mathcal{N} .
\end{aligned}
$$

Proof: This proof is similar to that for Theorem 1 and thus is omitted here for brevity.

Note that similar observations can be made from Corollary 1 as those in Remark 1. An additional tradeoff lies in designing the learning rate $\eta^{(n)}$. It is observed that when the learning rate is small, the error floor would be large as $C^{(n)}$ (or $J^{(n)}$ ) tends to be increasing, whereas the gap to the error floor becomes small.

\section{B. Optimality Gap versus Transmission Power Control}

In this subsection, we obtain the optimality gap w.r.t. the transmission power control variables based on the results in Section III-A in order to facilitate the power control design in the sequel. In particular, we consider the Air-FEEL in the cases without and with unbiased aggregation constraints $\mathbb{E}\left[\varepsilon^{(n)}\right]=$ $0, \forall n \in \mathcal{N}$.

Before proceeding, we introduce the following assumption on the sample-wise gradient bound.

Assumption 4 (Bounded sample-wise gradient). At any communication round $n$, the sample-wise gradient $\nabla f\left(\mathbf{w}^{(n)}, \mathbf{x}, y\right)$ for any training sample $(\mathbf{x}, y)$ is upper bounded by a given constant $G^{(n)}$, i.e.,

$$
\left\|\nabla f\left(\mathbf{w}^{(n)}, \mathbf{x}, y\right)\right\| \leq G^{(n)}, \forall n \in \mathcal{N} .
$$

Based on Assumption 4, we have $\left\|\nabla F\left(\mathbf{w}^{(n)}\right)\right\| \leq$ $\max _{(\mathbf{x}, y) \in \mathcal{D}}\left\|\nabla f\left(\mathbf{w}^{(n)}, \mathbf{x}, y\right)\right\| \leq G^{(n)}$. Together with Assumption 3 , it thus holds that

$$
\begin{aligned}
\mathbb{E}\left[\left\|\mathbf{g}_{k}^{(n)}\right\|^{2}\right] & \leq\left\|\nabla F\left(\mathbf{w}^{(n)}\right)\right\|^{2}+\frac{\|\boldsymbol{\sigma}\|_{2}^{2}}{m_{b}} \\
& \leq \hat{G}^{(n)} \triangleq\left(G^{(n)}\right)^{2}+\frac{\|\boldsymbol{\sigma}\|_{2}^{2}}{m_{b}} .
\end{aligned}
$$

1) Convergence Analysis for Air-FEEL in Case I: In this part, we formally characterize the convergence behavior of 
Air-FEEL w.r.t. the transmission power in the case without unbiased aggregation constraints.

According to the definition of $\varepsilon^{(n)}$ in formula (12), at each communication round $n$, the bias and MSE of gradient estimates through the over-the-air gradient aggregation for AirFEEL are derived as

$$
\begin{gathered}
\left\|\mathbb{E}\left[\varepsilon^{(n)}\right]\right\|=\frac{\left\|\nabla F\left(\mathbf{w}^{(n)}\right)\right\|}{K}\left(\sum_{k \in \mathcal{K}} h_{k}^{(n)} \sqrt{p_{k}^{(n)}}-K\right) \\
\leq \frac{G^{(n)}}{K}\left(\sum_{k \in \mathcal{K}} h_{k}^{(n)} \sqrt{p_{k}^{(n)}}-K\right) \\
\mathbb{E}\left[\left\|\varepsilon^{(n)}\right\|^{2}\right] \leq \\
\leq \frac{\left\|\nabla F\left(\mathbf{w}^{(n)}\right)\right\|^{2}+\frac{\|\boldsymbol{\sigma}\|_{2}^{2}}{m_{b}}}{K} \sum_{k \in \mathcal{K}}\left(h_{k}^{(n)} \sqrt{p_{k}^{(n)}}-1\right)^{2}+\frac{\sigma_{z}^{2} q}{K^{2}} \\
\leq \hat{G}^{(n)} \sum_{k \in \mathcal{K}}\left(h_{k}^{(n)} \sqrt{p_{k}^{(n)}}-1\right)^{2}+\frac{\sigma_{z}^{2} q}{K^{2}}
\end{gathered}
$$

where both inequalities follow from Assumptions 3 and 4 , By substituting (20) and (21) into (17) and (16), we can have the following proposition.

Proposition 1 (Optimality gap for Air-FEEL without unbiased aggregation constraints). The expected optimality gap for AirFEEL in the case without unbiased aggregation constraints is upper bounded by

$$
\begin{aligned}
& \mathbb{E}\left[F\left(\mathbf{w}^{(N+1)}\right)\right]-F^{\star} \leq \prod_{n \in \mathcal{N}} C^{(n)}\left(\left[F\left(\mathbf{w}^{(1)}\right)\right]-F^{\star}\right) \\
& +\sum_{n=1}^{N} J^{(n)}\left(A^{(n)}\left(\sum_{k \in \mathcal{K}} h_{k}^{(n)} \sqrt{p_{k}^{(n)}}-K\right)^{2}+\frac{\left(\eta^{(n)}\right)^{2} L\|\boldsymbol{\sigma}\|_{2}^{2}}{2 m_{b} K^{2}}\right) \\
& +\sum_{n=1}^{N} J^{(n)}\left(B^{(n)} \sum_{k \in \mathcal{K}}\left(h_{k}^{(n)} \sqrt{p_{k}^{(n)}}-1\right)^{2}+\frac{\left(\eta^{(n)}\right)^{2} \sigma_{z}^{2} L q}{K^{2}}\right),(22)
\end{aligned}
$$

where $A^{(n)}=\frac{\left(1+\left(\eta^{(n)}\right)^{2} L^{2}\right)\left(G^{(n)}\right)^{2}}{K^{2}}, B^{(n)}=\frac{\left(\eta^{(n)}\right)^{2} L \hat{G}^{(n)}}{K}$, and $J^{(n)}=\frac{\prod_{i=n}^{N} C^{(i)}}{2 C^{(n)}}$ for the diminishing learning rates $\eta^{(n)}=$ $\frac{u}{n+v}, \forall n \in \mathcal{N}$, with $v>0, u>1 / \delta$, and $\eta^{(1)} \leq \frac{2}{2+L}$; while $A^{(n)}=\frac{\left(1+\eta^{2} L^{2}\right)\left(G^{(n)}\right)^{2}}{K^{2}}, B^{(n)}=\frac{\eta^{2} L \hat{G}^{(n)}}{K}$, and $J^{(n)}=\frac{C^{N-n}}{2}$ for the fixed learning rate with $\eta=\eta^{(n)}, \forall n \in \mathcal{N}$, with $0 \leq$ $\eta \leq \frac{2}{2+L} \leq \frac{1}{\delta}$.

2) Convergence Analysis for Air-FEEL in Case II: Next, we consider the case with unbiased aggregation constraints, where we have $\mathbb{E}\left[\varepsilon^{(n)}\right]=0, \forall n \in \mathcal{N}$. Similar to Proposition 1 we have the following proposition.

Proposition 2 (Optimality gap for Air-FEEL with unbiased aggregation constraints). The expected optimality gap for Air-FEEL in the case with unbiased aggregation constraints is upper bounded by

$$
\begin{aligned}
& \mathbb{E}\left[F\left(\mathbf{w}^{(N+1)}\right)\right]-F^{\star} \leq \prod_{n \in \mathcal{N}} C^{(n)}\left(\left[F\left(\mathbf{w}^{(1)}\right)\right]-F^{\star}\right) \\
& +\sum_{n=1}^{N} J^{(n)}\left(B^{(n)} \sum_{k \in \mathcal{K}}\left(h_{k}^{(n)} \sqrt{p_{k}^{(n)}}-1\right)^{2}+\frac{\left(\eta^{(n)}\right)^{2} \sigma_{z}^{2} L q}{K^{2}}\right) \\
& +\sum_{n=1}^{N} J^{(n)} \frac{\left(\eta^{(n)}\right)^{2} L\|\boldsymbol{\sigma}\|_{2}^{2}}{2 m_{b} K^{2}},
\end{aligned}
$$

where $B^{(n)}=\frac{\left(\eta^{(n)}\right)^{2} L \hat{G}^{(n)}}{K^{2}}$ and $J^{(n)}=\frac{\prod_{i=n}^{N} C^{(i)}}{2 C^{(n)}}$ for the diminishing learning rates $\eta^{(n)}=\frac{u}{n+v}, \forall n \in \mathcal{N}$, with $v>0, u>1 / \delta$, and $\eta^{(1)} \leq \frac{2}{2+L}$; while $B^{(n)}=\frac{\eta^{2} L \hat{G}^{(n)}}{K^{2}}$ and $J^{(n)}=\frac{C^{N-n}}{2}$ for the fixed learning rate with $\eta=\eta^{(n)}, \forall n \in$ $\mathcal{N}$, with $0 \leq \eta \leq \frac{2}{2+L} \leq \frac{1}{\delta}$.

Since the derived convergence results for both the cases of diminishing and fixed learning rates share similar form, the subsequent power control optimization will be presented targeting the case with diminishing learning rates only for brevity, while the yielded insights hold for both cases.

\section{Power Control Optimization}

Given the convergence results of Air-FEEL in the preceding section, we are now ready to present the power control optimization polices for speeding up the convergence rate in this section.

To start with, we first reformulate the power constraints in (13) and (14) by leveraging Assumption 4 and inequality (19) to avoid the requirement of non-causal gradient information $g_{k}^{(n)}$. Hence, the individual power constraints at each communication round and the entire training process are respectively reformulated as

$$
\begin{aligned}
& p_{k}^{(n)} \hat{G}^{(n)} \leq P_{k}^{\max }, \forall k \in \mathcal{K}, n \in \mathcal{N}, \\
& \frac{1}{N} \sum_{n \in \mathcal{N}} p_{k}^{(n)} \hat{G}^{(n)} \leq P_{k}^{\text {ave }}, \forall k \in \mathcal{K},
\end{aligned}
$$

where $P_{k}^{\max } \triangleq q \hat{P}_{k}^{\max }$ and $P_{k}^{\text {ave }} \triangleq q \hat{P}_{k}^{\text {ave }}, \forall k \in \mathcal{K}$, are defined for notational convenience.

\section{A. Power Control Optimization for Case I}

We start with the case I without unbiased aggregation constraints. Discarding the irrelevant terms in (22) in Proposition 1 (i.e., the terms related to the initial optimality gap $\mathbb{E}\left[F\left(\mathbf{w}^{(1)}\right)\right]-F^{\star}$, the gradient variance bound $\frac{\left(\eta^{(n)}\right)^{2} L\|\boldsymbol{\sigma}\|_{2}^{2}}{2 m_{b} K^{2}}$, and the noise power $\left.\frac{\left(\eta^{(n)}\right)^{2} \sigma_{z}^{2} L q}{K^{2}}\right)$ in Proposition 1, we denote $\tilde{\Phi}\left(\left\{p_{k}^{(n)}\right\}\right)$ in the following as the effective optimality gap to be optimized.

$$
\begin{aligned}
\tilde{\Phi}\left(\left\{p_{k}^{(n)}\right\}\right) \triangleq & \sum_{n=1}^{N} J^{(n)} A^{(n)}\left(\sum_{k \in \mathcal{K}} h_{k}^{(n)} \sqrt{p_{k}^{(n)}}-K\right)^{2} \\
& +\sum_{n=1}^{N} J^{(n)} B^{(n)} \sum_{k \in \mathcal{K}}\left(h_{k}^{(n)} \sqrt{p_{k}^{(n)}}-1\right)^{2} .
\end{aligned}
$$

The optimization problem is thus formulated as

$$
\begin{array}{cl}
\text { P1 : } \min _{\left\{p_{k}^{(n)} \geq 0\right\}} & \tilde{\Phi}\left(\left\{p_{k}^{(n)}\right\}\right) \\
\text { s.t. } & 24 \text { and 25. }
\end{array}
$$

By introducing a set of auxiliary variables, $\hat{p}_{k}^{(n)}=$ $\sqrt{p_{k}^{(n)}}, \forall k \in \mathcal{K}, n \in \mathcal{N}$, the objective is re-expressed as

$$
\begin{aligned}
\Phi\left(\left\{\hat{p}_{k}^{(n)}\right\}\right) \triangleq & \sum_{n=1}^{N} J^{(n)} A^{(n)}\left(\sum_{k \in \mathcal{K}} h_{k}^{(n)} \hat{p}_{k}^{(n)}-K\right)^{2} \\
& +\sum_{n=1}^{N} J^{(n)} B^{(n)} \sum_{k \in \mathcal{K}}\left(h_{k}^{(n)} \hat{p}_{k}^{(n)}-1\right)^{2},
\end{aligned}
$$


and problem (P1) is re-expressed as

$$
\begin{aligned}
\text { P1.1: } \min _{\left\{\hat{p}_{k}^{(n)} \geq 0\right\}} & \Phi\left(\left\{\hat{p}_{k}^{(n)}\right\}\right) \\
\text { s.t. } \quad & \hat{q}_{k}^{(n)} \leq P_{k, n}^{\max }, \forall k \in \mathcal{K}, n \in \mathcal{N} \\
& \frac{1}{N} \sum_{n \in \mathcal{N}}\left(\hat{q}_{k}^{(n)}\right)^{2} \hat{G}^{(n)} \leq P_{k}^{\text {ave }}, \forall k \in \mathcal{K},
\end{aligned}
$$

where constraints (28) and 29) follow from (24) and 25), respectively, and $P_{k, n}^{\max } \triangleq \sqrt{\frac{P_{k}^{\max }}{\hat{G}^{(n)}}}, \forall k \in \mathcal{K}, n \in \mathcal{N}$. Problem (P1.1) is convex and can thus be optimally solved by the standard convex optimization techniques such as the interior point method [50]. Alternatively, to gain engineering insights, we resort to the Lagrange duality method to derive the structured optimal solution for problem (P1.1). Let $\left\{\hat{p}_{k}^{(n) \text { opt }}\right\}$ denote the optimal solution to problem (P1.1), and $\varphi_{k}^{\text {opt }}, \forall k \in \mathcal{K}$ the optimal dual variable associated with the $k$-th constraint in (29). Then we have the following proposition.

Proposition 3. The optimal solution $\hat{p}_{k}^{(n) \text { opt }}, \forall k \in \mathcal{K}, n \in \mathcal{N}$ to problem $(\mathrm{P} 1.1)$ is

$$
\hat{p}_{k}^{(n) \mathrm{opt}}=\min \left[\frac{B^{(n)}+A^{(n)} K}{M_{k}^{(n)}+A^{(n)} M_{k}^{(n)} \sum_{i \in \mathcal{K}} \frac{h_{i}^{(n)}}{M_{i}^{(n)}}}, P_{k, n}^{\max }\right],
$$

where $M_{k}^{(n)} \triangleq B^{(n)} h_{k}^{(n)}+\frac{\varphi_{k}^{\mathrm{opt}} \hat{G}^{(n)}}{N J^{(n)} h_{k}^{(n)}}, \forall k \in \mathcal{K}, n \in \mathcal{N}$.

Proof: See Appendix B

According to Proposition 3, the optimal power scaling factors $p_{k}^{(n) \text { opt }}, \forall k \in \mathcal{K}, n \in \mathcal{N}$ to problem (P1) is

$p_{k}^{(n) \mathrm{opt}}=\min \left[\left(\frac{B^{(n)}+A^{(n)} K}{M_{k}^{(n)}+A^{(n)} M_{k}^{(n)} \sum_{i \in \mathcal{K}} \frac{h_{i}^{(n)}}{M_{i}^{(n)}}}\right)^{2},\left(P_{k, n}^{\max }\right)^{2}\right]$.

Remark 2. According to Proposition 3, the optimal $\left\{\hat{p}_{k}^{(n) \text { opt }}\right\}$ (equivalently the optimal power scaling factor $p_{k}^{(n) \text { opt }}=$ $\left.\left(\hat{p}_{k}^{(n) \text { opt }}\right)^{2}, \forall k \in \mathcal{K}, n \in \mathcal{N}\right)$ exhibits a regularized channel inversion structure with the regularized term $\sum_{i \in \mathcal{K}} \frac{A^{(n)} h_{i}^{(n)} M_{k}^{(n)}}{M_{i}^{(n)}}$ related to all dual variables $\varphi_{k}^{\text {opt }}$ associated with the average power budgets at all edge devices in 29. Considering the special case when the average power budgets $\left\{P_{k}^{\text {ave }}\right\}$ at all devices are sufficiently large, such that the dual variables become zero at the same time (i.e., $\varphi_{k}^{\text {opt }}=0, \forall k \in \mathcal{K}, n \in \mathcal{N}$ ). In this case, the optimal power scaling strategy reduces to the channel inversion policy, i.e., $p_{k}^{(n) \text { opt }}=\min \left[\frac{1}{\left(h_{k}^{(n)}\right)^{2}},\left(P_{k, n}^{\max }\right)^{2}\right]$, $\forall k \in \mathcal{K}, n \in \mathcal{N}$. Interestingly, this result is equivalent to minimizing the MSE in isolation at each communication round. In other words, in the special case when all devices have a sufficiently large average power budgets, the conventional MSE minimization can be sufficient to minimize the optimality gap.

\section{B. Power Control Optimization fo Case II}

Next, we consider the power control optimization for the case with unbiased aggregation constraints, where the power control policy needs to enforce the additional constraint $\mathbb{E}\left[\varepsilon^{(n)}\right]=0, \forall n \in \mathcal{N}$. According to (20), it follows that $\sum_{k \in \mathcal{K}} h_{k}^{(n)} \sqrt{p_{k}^{(n)}}=K, \forall n \in \mathcal{N}$. In this case, the effective optimality gap in Proposition 2 is given by

$$
\tilde{\Theta}\left(\left\{p_{k}^{(n)}\right\}\right) \triangleq \sum_{n=1}^{N} J^{(n)} B^{(n)} \sum_{k \in \mathcal{K}}\left(h_{k}^{(n)} \sqrt{p_{k}^{(n)}}-1\right)^{2} .
$$

Accordingly, we formulate the power control optimization problem as

$$
\begin{aligned}
\text { P2: } \min _{\left\{p_{k}^{(n)} \geq 0\right\}} & \tilde{\Theta}\left(\left\{p_{k}^{(n)}\right\}\right) \\
\text { s.t. } & \sum_{k \in \mathcal{K}} h_{k}^{(n)} \sqrt{p_{k}^{(n)}}=K, \forall n \in \mathcal{N} \\
& \text { 24) and 25. }
\end{aligned}
$$

Note that problem (P2) is non-convex. However, via a change of variables $q_{k}^{(n)} \triangleq \sqrt{p_{k}^{(n)}}, \forall k \in \mathcal{K}, n \in \mathcal{N}$, the objective can be re-expressed as

$$
\Theta\left(\left\{q_{k}^{(n)}\right\}\right) \triangleq \sum_{n=1}^{N} J^{(n)} B^{(n)} \sum_{k \in \mathcal{K}}\left(h_{k}^{(n)} q_{k}^{(n)}-1\right)^{2},
$$

and problem $(\mathrm{P} 2)$ can be transformed into the following equivalent convex form:

$$
\begin{aligned}
\text { P2.1 : } \min _{\left\{q_{k}^{(n)} \geq 0\right\}} \Theta\left(\left\{q_{k}^{(n)}\right\}\right) \\
\text { s.t. } \quad \sum_{k \in \mathcal{K}} h_{k}^{(n)} q_{k}^{(n)}=K, \forall n \in \mathcal{N} \\
\\
q_{k}^{(n)} \leq P_{k, n}^{\max }, \forall k \in \mathcal{K}, \forall n \in \mathcal{N} \\
\frac{1}{N} \sum_{n \in \mathcal{N}}\left(q_{k}^{(n)}\right)^{2} \hat{G}^{(n)} \leq P_{k}^{\text {ave }}, \forall k \in \mathcal{K},
\end{aligned}
$$

where constraints (36) and (37) follow from (24) and (25), respectively.

1) Feasibility of Problem (P2.1): Before solving problem (P2.1), we first check its feasibility, i.e., whether the power budget can support the required unbiased estimation level denoted by $\ell$ or not. Let $\ell^{\star}$ denote the maximum unbiased estimation level, which can be expressed as

$$
\begin{aligned}
\ell^{\star}=\max _{\left\{q_{k}^{(n)} \geq 0\right\}} & \ell \\
\text { s.t. } & \sum_{k \in \mathcal{K}} h_{k}^{(n)} q_{k}^{(n)} \geq \ell, \forall n \in \mathcal{N}
\end{aligned}
$$$$
\text { (36) and (37). }
$$

If $\ell^{\star} \geq K$, then problem ( $\left.\mathrm{P} 2.1\right)$ is feasible; otherwise, problem $(\mathrm{P} 2.1)$ is not feasible. Hence, the feasibility checking procedure corresponds to finding $\ell^{\star}$ by solving problem (38). Notice that problem (38) is convex, which can thus be efficiently solved via standard convex optimization techniques, such as the interior point method [50]. By comparing $\ell^{\star}$ versus $K$, the feasibility of problem (P2.1) is checked. In the following, we solve problem (P2.1) when it is feasible.

2) Optimal Solution to Problem (P2.1): Let $\left\{q_{k}^{(n) \text { opt }}\right\}$ denote the optimal solution to problem $(\mathrm{P} 2.1)$. We have the following proposition by leveraging the Lagrange duality method, where $\mu_{n}^{\text {opt }}$ and $\lambda_{k}^{\text {opt }}$ are the optimal dual variables associated with constraints (35) and (37), respectively. 
Proposition 4. The optimal solution $q_{k}^{(n) \mathrm{opt}}, \forall k \in \mathcal{K}, n \in \mathcal{N}$ to problem (P2.1) is given as

$$
q_{k}^{(n) \text { opt }}=\min \left[\frac{h_{k}^{(n)} \alpha_{k}^{(n)}}{\left(h_{k}^{(n)}\right)^{2}+\frac{2 \lambda_{k}^{\text {opt }}}{N J^{(n)} B^{(n)}}}, P_{k, n}^{\max }\right],
$$

where $\alpha_{k}^{(n)} \triangleq\left(1-\frac{\mu_{n}^{\mathrm{opt}}}{2 J^{(n)} B^{(n)}}\right)^{+}, \forall k \in \mathcal{K}, n \in \mathcal{N}$.

Proof: See Appendix C

From (39) in Proposition 4 we can accordingly obtain the optimal power scaling factors $p_{k}^{(n) \text { opt }}, \forall k \in \mathcal{K}, n \in \mathcal{N}$ to problem (P2) as

$$
\begin{aligned}
p_{k}^{(n) \mathrm{opt}} & =\left(q_{k}^{(n) \mathrm{opt}}\right)^{2} \\
& =\min \left[\left(\frac{h_{k}^{(n)} \alpha_{k}^{(n)}}{\left(h_{k}^{(n)}\right)^{2}+\frac{2 \lambda_{k}^{\mathrm{opt}} \hat{G}^{(n)}}{N J^{(n)} B^{(n)}}}\right)^{2},\left(P_{k, n}^{\max }\right)^{2}\right] .
\end{aligned}
$$

Remark 3. According to (39), the optimal solution of $\left\{q_{k}^{(n) \text { opt }}\right\}$ to problem (P2.1) (equivalently the optimal power scaling factor $\left.p_{k}^{(n) \text { opt }}=\left(q_{k}^{(n) \text { opt }}\right)^{2}, \forall k \in \mathcal{K}, n \in \mathcal{N}\right)$ has a similar regularized channel inversion structure as that in (30), but the regularized term therein (i.e., $\frac{2 \lambda_{k}^{\text {opt }} \hat{G}^{(n)}}{N J^{(n)} B^{(n)}}$ ) is only related to its own device $k$ 's average power budget in 37) through the dual variable $\lambda_{k}^{\text {opt }}$, as opposed to all devices' budgets in 29) for the case without unbiased aggregation constraints. Furthermore, it is observed that for any edge device $k \in \mathcal{K}$, if $\lambda_{k}^{\text {opt }}>0$ holds, then the average power constraint of edge device $k$ must be tight at the optimality (i.e., $\left.\frac{1}{N} \sum_{n \in \mathcal{N}}\left(q_{k}^{(n) \text { opt }}\right)^{2} \hat{G}^{(n)}-P_{k}^{\text {ave }}=0\right)$ due to the complementary slackness condition, and thus this edge device should use up its average power budget based on the regularized channel inversion power control over communication rounds; otherwise, if $\lambda_{k}^{\mathrm{opt}}=0$, then edge device $k$ should transmit with channelinversion power control without using up its average power budget.

\section{Simulation Results}

In this section, we provide simulation results to validate the performance of the proposed power control policies for Air-FEEL. The proposed algorithms are implemented using the Matlab and Pytorch for two different tasks, i.e., the ridge regression and handwritten digit recognition, respectively.

\section{A. Simulation Setup and Benchmark Schemes}

In the simulation, the wireless channels from the edge devices to the edge server over different communication rounds follow i.i.d. Rayleigh fading, i.e., $h_{k}^{(n)}$, s are modeled as i.i.d. circularly symmetric complex Gaussian (CSCG) random variables with zero mean and unit variance. We set the number of devices as $K=10$, the noise variance $\sigma_{z}^{2}=0.1$, and the average power budgets at different devices $\hat{P}_{k}^{\text {ave }}$ to be heterogeneou 4 . We set the maximum power budget to be $5 \hat{P}^{\text {ave }}$. We consider both the fixed and diminishing learning

\footnotetext{
${ }^{4}$ The average power budgets at different devices are set as, $\hat{P}_{i}^{\text {ave }}=5 \mathrm{~W}$ and $\hat{P}_{i+1}^{\text {ave }}=15 \mathrm{~W}, i=\{1, \cdots, K / 2\}$.
}

rates with $\eta=0.05$ and $\eta^{(n)}=\frac{u}{n+v}$ under $u=2$ and $v=8$, respectively. As for the performance metrics, the optimality gap and prediction error are considered for ridge regression on synthetic dataset, while the loss function value and test (recognition) accuracy are considered for handwritten digit recognition on MNIST dataset.

For performance comparison, we consider the following two benchmark schemes.

- Fixed power transmission: The edge devices transmit with fixed power over different communication rounds by setting $p_{k}^{(n)}=P_{k}^{\text {ave }}, \forall k \in \mathcal{K}$.

- Conventional MSE minimization: The edge devices optimize their power control to minimize the aggregation MSE in isolation at each communication round. For each round, the MSE minimization problem has been solved in $[15]$.

\section{B. Air-FEEL for Ridge Regression}

First, we consider the ridge regression with the samplewise loss function $f(\mathbf{w}, \mathbf{x}, \tau)=\frac{1}{2}\left\|\mathbf{x}^{T} \mathbf{w}-\tau\right\|^{2}+\rho R(\mathbf{w})$ 6 and the regularization function $R(\mathbf{w})=\|\mathbf{w}\|^{2}$ with the hyperparameter $\rho=5 \times 10^{-5}$. Randoml,y generated synthetic dataset is used for model training and testing. The generated data sample vector $\mathbf{x} \in \mathbb{R}^{q}$ with $q=10$ follow i.i.d. Gaussian distribution (i.e., $x \sim \mathcal{N}(0, \mathbf{I})$ ) and the label $y$ is obtained as $\tau=x(2)+3 x(5)+0.2 z$, where $x(t)$ represents the $t$-th in vector $\mathbf{x}$ and $z$ denotes the observation noise with i.i.d. Gaussian distribution, i.e., $z \sim \mathcal{N}(0,1)$. Unless stated otherwise, the data samples are evenly distributed among devices with identical size $D=D_{k}=1000, \forall k \in \mathcal{K}$ and $D_{\text {tot }}=\sum_{k \in \mathcal{K}} D_{k}=10000$.

Based on the above models, we can obtain the smoothness parameter $L$ and Polyak-Łojasiewicz parameter $\delta$ as the largest and smallest eigenvalues of the data Gramian matrix $\mathbf{X}^{T} \mathbf{X} / D_{\text {tot }}+10^{-4} \mathbf{I}$, in which $\mathbf{X}=\left[\mathbf{x}_{1}, \cdots, \mathbf{x}_{D_{\text {tot }}}\right]^{T}$ is the data matrix. We use the simple upper bounds $G^{(n)}=2 W L$ [37] with $W \geq\|\mathbf{w}\|$ as a bound on the norm $\|\mathbf{w}\|$. The optimal loss function $F^{\star}$ is computed using the optimal parameter vector $\mathbf{w}^{\star}$ to the learning problem (3), where $\mathbf{w}^{\star}=\left(\mathbf{X}^{T} \mathbf{X}+\rho \mathbf{I}\right)^{-1} \mathbf{X}^{T} \boldsymbol{\tau}$ with $\boldsymbol{\tau}=\left[\tau_{1}, \cdots, \tau_{D_{\mathrm{tot}}}\right]^{T}$. We set the initial parameter vector as an all-zero vector.

Fig. 3 shows the learning performance (i.e., the optimality gap in Figs. 3(a) and 3(c) and the prediction error in Figs. 3(b) and 3(d) versus the number of communication rounds $N$, where the learning rates are set to be diminishing in Figs. 3(a) and 3(b) and those are set to be fixed in Figs. 3(c) and 3(d), First, it is observed that the proposed

\footnotetext{
${ }^{5}$ Although the conventional channel inversion power control can achieve the unbiased aggregation, it is not the only way to achieve the unbiased aggregation and just a sufficient condition leading to unbiased aggregation. Moreover, as validated in [15], the conventional MSE minimization scheme can achieve the minimum communication distortion in AirComp. Therefore, in this paper we only consider the conventional MSE minimization scheme as one benchmark, which always outperforms the generally sub-optimal channel inversion scheme.

${ }^{6}$ The loss function here consists of both the model loss and the regularization term, where the former captures the prediction error of the trained model over the samples, and the latter is added to avoid overfitting and enhance the robustness [51].
} 


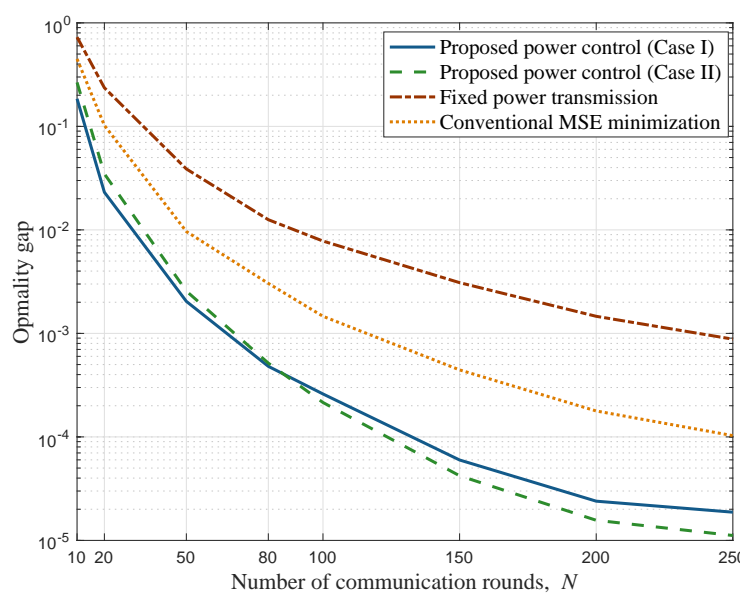

(a) Optimality gap versus $N$ under diminishing learning rate.

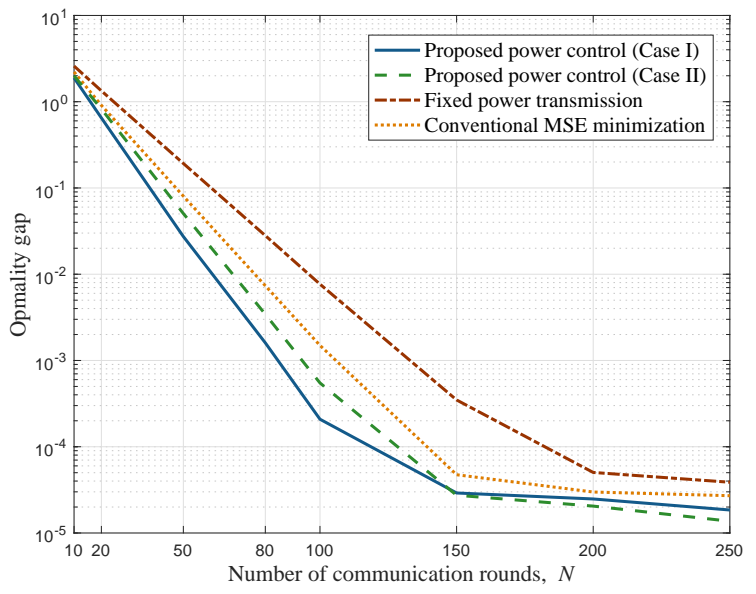

(c) Optimality gap versus $N$ under fixed learning rate.

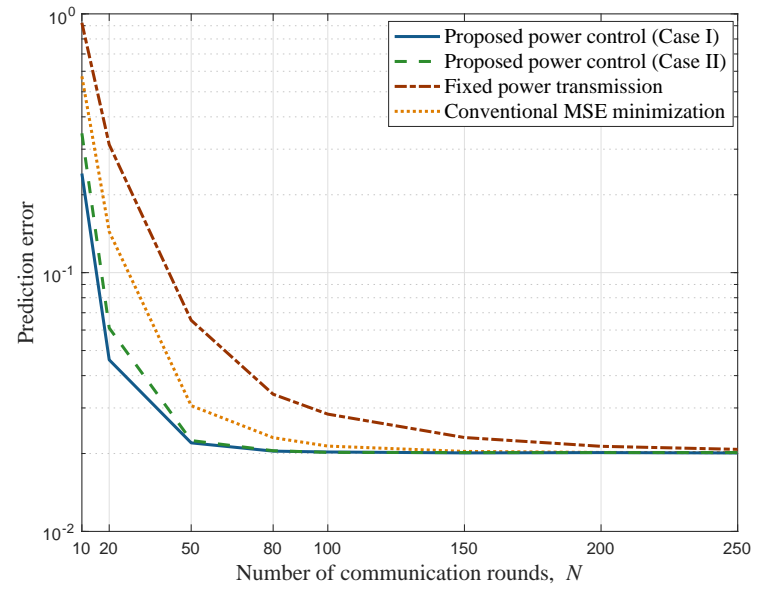

(b) Prediction error versus $N$ under diminishing learning rate.

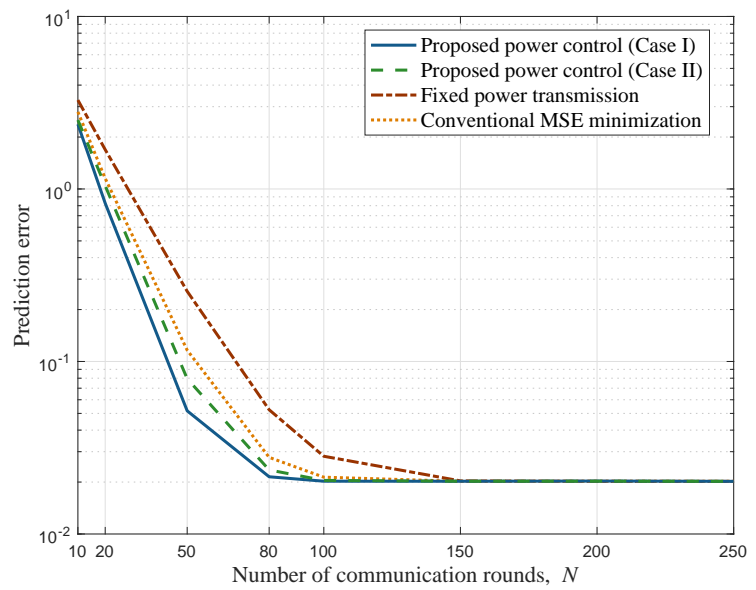

(d) Prediction error versus $N$ under fixed learning rate.

Figure 3. Learning performance of Air-FEEL over number of communication rounds.

power control policies (with both biased and unbiased aggregation constraints) and the conventional MSE minimization design achieve faster convergence and lower optimality gap than the fixed power transmission. This shows the benefit of power control optimization in accelerating the learning convergence rate, via either directly minimizing the optimality gap or indirectly minimizing the MSE. Secondly, the proposed power control policies are observed to significantly outperform the conventional MSE minimization design in reducing the optimality gap. This is due to the fact that the contributions of aggregation errors to the optimality gap are distinct at different communication rounds (see Remark 1), which cannot be captured by the conventional MSE minimization design. Furthermore, the proposed power control policy under Case II (with unbiased aggregation constraints) is observed to achieve a lower optimality gap than the proposed power control policy under Case I (without unbiased aggregation constraints) when $N>150$ under the fixed learning rate and $N>80$ under the diminishing learning rates. This coincides with Remark 1 that the Air-FEEL algorithm converges to the optimal point with unbiased gradient aggregation.

Fig. 4 shows the learning performance (i.e., the optimality gap in Figs. 4(a) and 4(c) and the prediction error in Figs. 4(b) and 4(d) $)$ versus the number of devices $K$, where the learning rates are set to be diminishing in Figs. 4(a) and 4(b) and those are set to be fixed in Figs. 4(c) and 4(d) Firstly, it is observed that the optimality gap achieved by all schemes decreases as $K$ increases. This is because that the edge server can aggregate more data for averaging to improve the learning performance. Secondly, the performance gaps between the proposed power control policies (under Cases I and II) versus the benchmark schemes are observed to decrease with $K$ increasing, which would be saturated in the large $K$ regime. The performance gap validates the effectiveness on the proposed power control optimization in reducing the optimality gap.

\section{Air-FEEL for Handwritten Digit Recognition}

Next, we consider the learning task of handwritten digit recognition using the well-known MNIST datasets, which consists of 10 classes of black-and-white digits ranging from " 0 " to "9". We implement a 6-layer CNN as the classifier model, which consists of two $5 \times 5$ convolution layers with ReLU activation (the first with 32 channels, the second with 64 ), each followed by a $2 \times 2$ max pooling; a fully connected layer with 512 units and ReLU activation; and a final softmax output layer $(582,026$ parameter in total). The local batch 


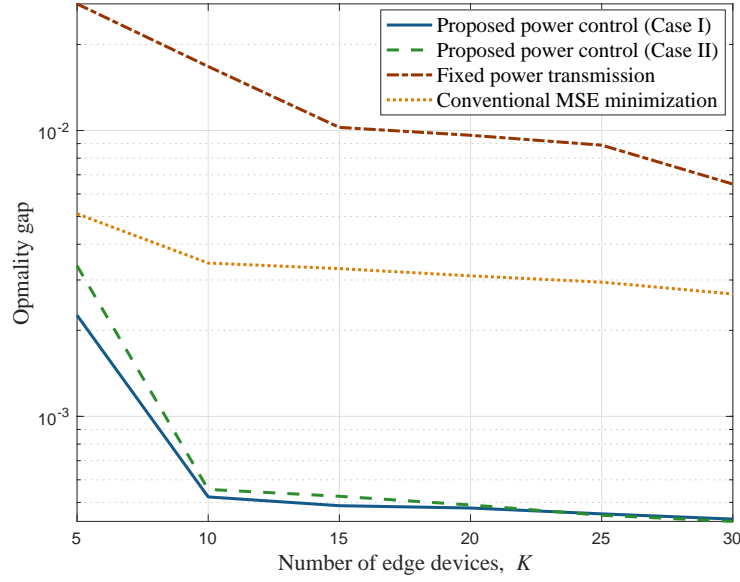

(a) Optimality gap versus $K$ under diminishing learning rate.

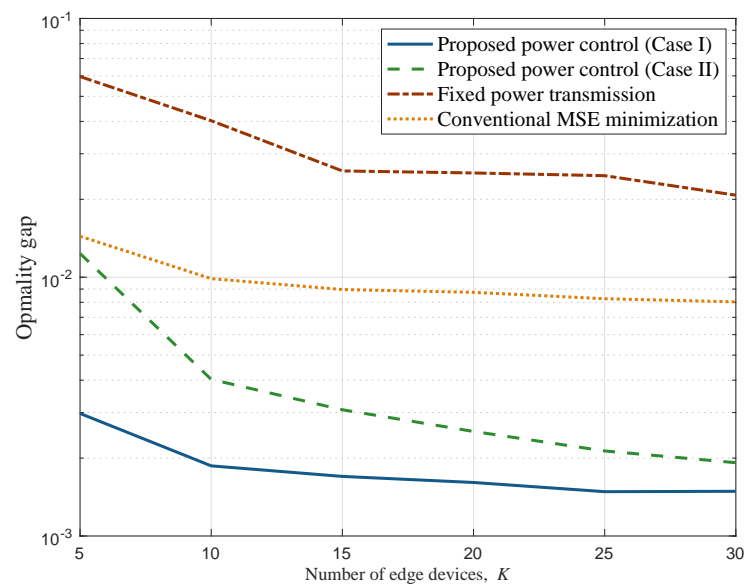

(c) Optimality gap versus $K$ under fixed learning rate.

Figure 4. Effect of number of devices on the learning performance of Air-FEEL.

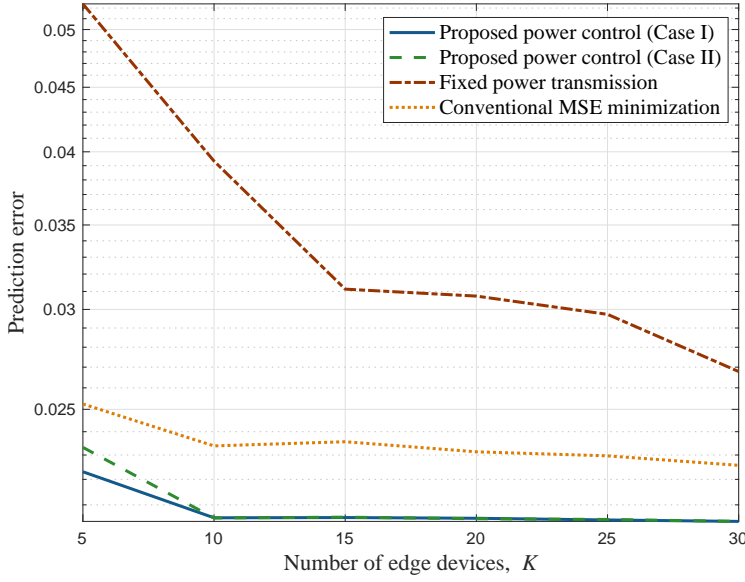

(b) Prediction error versus $K$ under diminishing learning rate

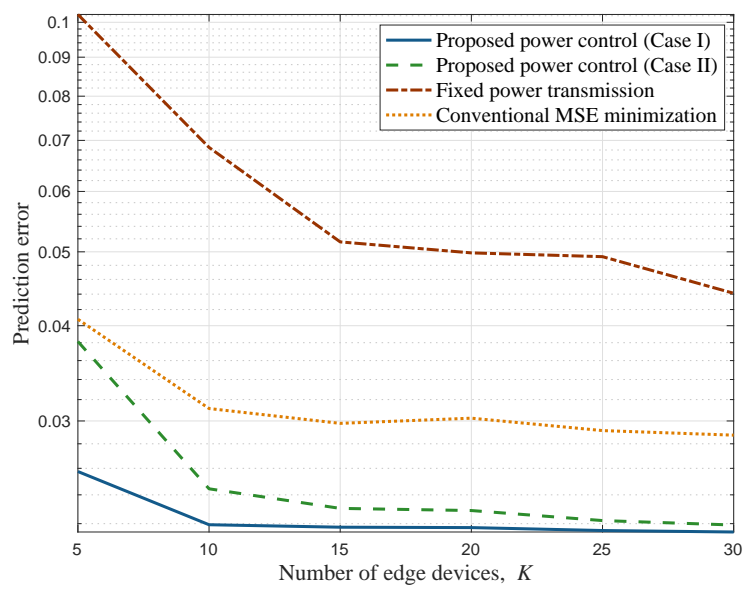

(d) Prediction error versus $K$ under fixed learning rate. size at each edge device is set to be $m_{b}=512$. Notice that Assumptions 11 and 2 may not hold in this case, but our proposed power control policies still work well as will be shown shortly.

Fig. 5 shows the learning performance versus the varying number of communication rounds $N$, where the learning rates are set to be diminishing in Figs. 5(a) and 5(b) and those are set to be fixed with $\eta=0.01$ in Figs. 5(c) and 5(d) First, it is observed that the proposed power control policies achieve lower loss function values and higher test accuracy than both the fixed-power-transmission and conventionalMSE-minimization schemes. Furthermore, the power control policy under Case II is observed to outperform that under Case I when $N>200$ with the fixed learning rate and $N>150$ with the diminishing learning rates. These observations are generally consistent with those in Fig. 3 with the ridge regression model.

\section{CONCLUSION}

In this paper, we exploited the transmission power control as a new design degree of freedom to optimize the learning performance of Air-FEEL. To this end, we first analyzed the convergence behavior of the FEEL algorithm (in terms of the optimality gap) and characterized the impact of aggregation errors, w.r.t. its bias and MSE, at different communication rounds. It was observed that in the case with unbiased aggregation estimates, the FEEL algorithm would converge exactly to the optimal point with mild conditions; and otherwise, it would converge with an error floor. Next, we proposed to directly minimize the derived optimality gaps by optimizing the power control, for which the optimal solutions are obtained to follow regularized channel inversion structures. Finally, experimental results demonstrated that the proposed power control policies achieve significantly lower optimality gap in Air-FEEL, as compared with benchmark schemes with fixed power transmission and conventional MSE minimization. We expect that this initial work can provide useful insights on exploiting the power control for enhancing the Air-FEEL performance.

Nevertheless, due to the space limitation, there are still a lot of interesting issues that are not addressed in this paper but worth investigation. In the following, we introduce several of them to motivate future work.

- One interesting direction is to explore the large-scale AirFEEL over multi-cell networks to accommodate massive edge devices with more data. In this case, a hierar- 


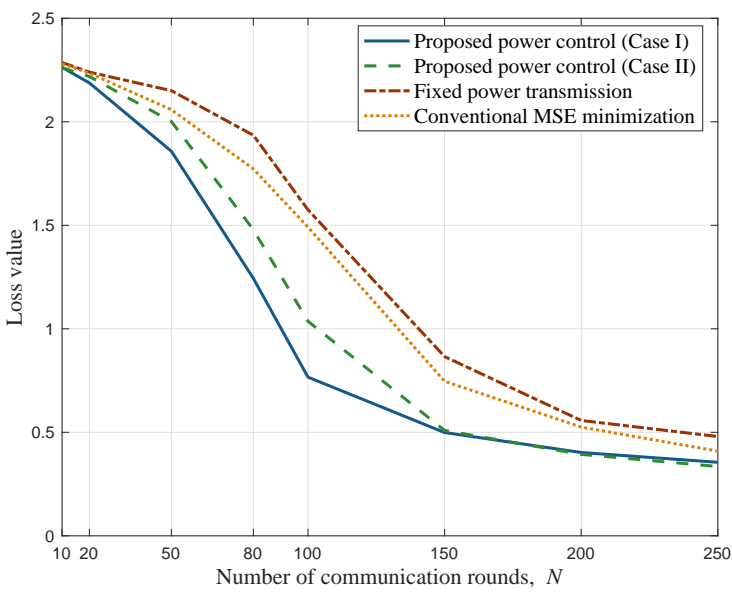

(a) Loss value versus $N$ under diminishing learning rate.

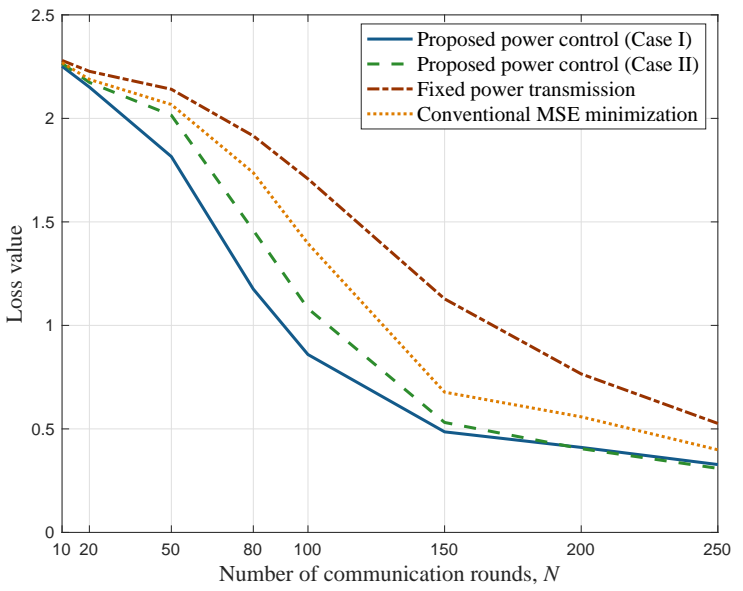

(c) Loss value versus $N$ under fixed learning rate.

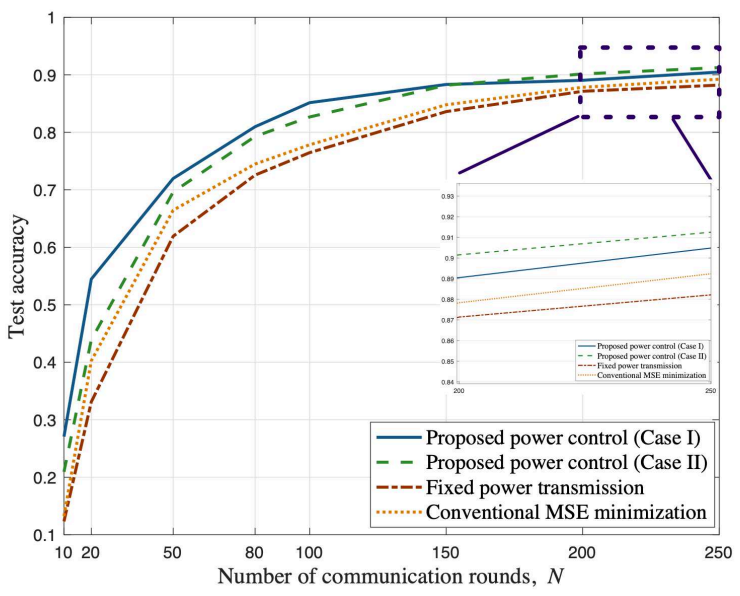

(b) Test accuracy versus $N$ under diminishing learning rate.

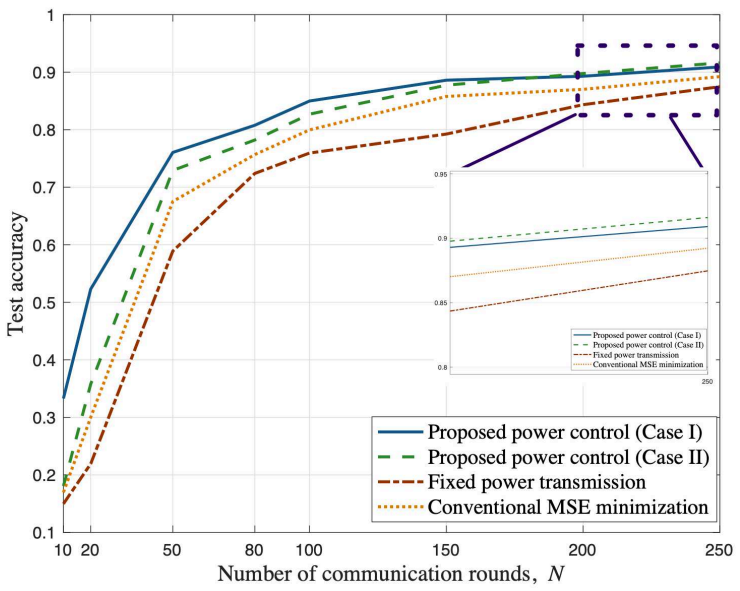

(d) Test accuracy versus $N$ under fixed learning rate.

Figure 5. Learning performance of Air-FEEL on MNIST dataset over number of communication rounds.

chical framework would be established to explore the distributed computation and communication capacity for performance improvement, and therein the cooperative interference management will be a new issue to be addressed.

- Another interesting direction is to consider the FedAvg scenario, where the local updates at each edge devices are implemented multiple times, and the local models are aggregated over the air instead of gradients. The analysis and design principles in this paper are generally extendable to this scenario by taking into account the following two new technical challenges. First, as multiple local updates are implemented at edge devices, the introduced gradient/model errors due to aggregation in this paper are not applicable for FedAvg. Therefore, new approaches for analyzing the gradient/model errors in FedAvg should be considered for its convergence analysis. Next, while the learning rate plays a dual role of denoising factor for over-the-air gradient aggregation in FedSGD, in FedAvg we need a dedicated denoising factor to suppress the AirComp signal misalignment error for model aggregation. As a result, the denoising factors would become new design variables for joint optimization, thus making the problem more complicated.

- Furthermore, it is also an interesting problem to fairly and quantitatively compare the performance of Air-FEEL versus the traditional digital FEEL (e.g., [5], [7], [9]), in terms of the training latency and training accuracy. General speaking, the proposed Air-FEEL can achieve significant per-round communication latency reduction over the digital FEEL, but at a cost of the newly introduced aggregation errors and the increased number of communication rounds needed for convergence. To deal with such a tradeoff, we need to explore the power control policy for training latency minimization while ensuring a given maximum optimality gap requirement for both Air-FEEL and digital FEEL. How to determine proper approximate optimality gaps for both Air-FEEL and digital FEEL to enable their fair training performance comparison is challenging in practice.

- Moreover, the investigation of Air-FEEL with the noni.i.d. data is also an interesting future research direction. The non-i.i.d. nature of data may degrade the training performance of AirFEEL, and also make the convergence analysis more challenging. In this case, how to capture the effect of the non-i.i.d. degrees of edge devices on 
the learning performance and accordingly optimize the transmission power control over them is a difficult task.

\section{APPENDIX}

\section{A. Proof of Theorem 1}

The proof follows by relating the norm of the gradient to the expected improvement made at each communication round. Recall that $\mathbf{w}^{(n+1)}=\mathbf{w}^{(n)}-\eta \cdot\left(\overline{\mathbf{g}}^{(n)}+\varepsilon^{(n)}\right)$, and thus it follows that

$$
\begin{aligned}
F & \left(\mathbf{w}^{(n+1)}\right)-F\left(\mathbf{w}^{(n)}\right) \\
\leq & \left(\nabla F\left(\mathbf{w}^{(n)}\right)\right)^{T}\left(\mathbf{w}^{(n+1)}-\mathbf{w}^{(n)}\right)+\sum_{i=1}^{q} \frac{L_{i}}{2}\left(w_{i}^{(n+1)}-w_{i}^{(n)}\right)^{2} \\
= & \nabla F\left(\mathbf{w}^{(n)}\right)^{T}\left(\mathbf{w}^{(n)}-\eta \cdot\left(\overline{\mathbf{g}}^{(n)}+\boldsymbol{\varepsilon}^{(n)}\right)-\mathbf{w}^{(n)}\right) \\
& +\frac{L}{2}\left\|\mathbf{w}^{(n)}-\eta \cdot\left(\overline{\mathbf{g}}^{(n)}+\boldsymbol{\varepsilon}^{(n)}\right)-\mathbf{w}^{(n)}\right\|^{2} \\
= & -\eta \nabla F\left(\mathbf{w}^{(n)}\right)^{T}\left(\overline{\mathbf{g}}^{(n)}+\boldsymbol{\varepsilon}^{(n)}\right)+\frac{L \eta^{2}}{2}\left\|\overline{\mathbf{g}}^{(n)}+\boldsymbol{\varepsilon}^{(n)}\right\|^{2},(41)
\end{aligned}
$$

where the above inequality follows Assumption 1 and $L \triangleq$ $\|\mathbf{L}\|_{\infty}$. By taking expectation at both sides of 4 41), we have $\mathbb{E}\left[F\left(\mathbf{w}^{(n+1)}\right)-F\left(\mathbf{w}^{(n)}\right)\right]$

$$
\begin{aligned}
\leq & -\eta \nabla F\left(\mathbf{w}^{(n)}\right)^{T} \mathbb{E}\left[\overline{\mathbf{g}}^{(n)}+\boldsymbol{\varepsilon}^{(n)}\right]+\frac{L \eta^{2}}{2} \mathbb{E}\left[\left\|\overline{\mathbf{g}}^{(n)}+\boldsymbol{\varepsilon}^{(n)}\right\|_{2}^{2}\right] \\
= & -\eta\left\|\nabla F\left(\mathbf{w}^{(n)}\right)\right\|^{2}-\eta \nabla F\left(\mathbf{w}^{(n)}\right)^{T} \mathbb{E}\left[\varepsilon^{(n)}\right]+\frac{L \eta^{2}}{2} \mathbb{E}\left[\left\|\overline{\mathbf{g}}^{(n)}\right\|^{2}\right] \\
& +L \eta^{2} \nabla F\left(\mathbf{w}^{(n)}\right)^{T} \mathbb{E}\left[\varepsilon^{(n)}\right]+\frac{L \eta^{2}}{2} \mathbb{E}\left[\left\|\varepsilon^{(n)}\right\|^{2}\right] \\
\leq & -\eta\left\|\nabla F\left(\mathbf{w}^{(n)}\right)\right\|^{2}-\eta \nabla F\left(\mathbf{w}^{(n)}\right)^{T} \mathbb{E}\left[\varepsilon^{(n)}\right] \\
& +\frac{L \eta^{2}}{2}\left(\frac{\left\|\nabla F\left(\mathbf{w}^{(n)}\right)\right\|_{2}^{2}}{K}+\frac{\|\boldsymbol{\sigma}\|_{2}^{2}}{m_{b} K^{2}}\right) \\
& +\frac{L \eta^{2}}{2} \mathbb{E}\left[\left\|\varepsilon^{(n)}\right\|^{2}\right]+L \eta^{2} \nabla F\left(\mathbf{w}^{(n)}\right)^{T} \mathbb{E}\left[\varepsilon^{(n)}\right],
\end{aligned}
$$

where the denominator $m_{b}$ in (42) is induced from Assumption 3 and Equation (4). By applying the inequality of arithmetic and geometric means, i.e., $-\boldsymbol{a}_{1}{ }^{T} \boldsymbol{a}_{2} \leq \frac{\left\|\boldsymbol{a}_{1}\right\|^{2}}{2}+\frac{\left\|\boldsymbol{a}_{2}\right\|^{2}}{2}$, it then follows that

$$
\begin{aligned}
& \mathbb{E}\left[F\left(\mathbf{w}^{(n+1)}\right)-F\left(\mathbf{w}^{(n)}\right)\right] \\
& \leq-\eta\left\|\nabla F\left(\mathbf{w}^{(n)}\right)\right\|^{2}+\frac{\eta^{2}}{2}\left\|\nabla F\left(\mathbf{w}^{(n)}\right)\right\|^{2}+\frac{\left\|\mathbb{E}\left[\varepsilon^{(n)}\right]\right\|^{2}}{2} \\
& \quad+\frac{L \eta^{2}}{2} \mathbb{E}\left[\left\|\varepsilon^{(n)}\right\|^{2}\right]+L \eta^{2} \nabla F\left(\mathbf{w}^{(n)}\right)^{T} \mathbb{E}\left[\varepsilon^{(n)}\right] \\
& \quad+\frac{L \eta^{2}}{2}\left(\left\|\nabla F\left(\mathbf{w}^{(n)}\right)\right\|_{2}^{2}+\frac{\|\boldsymbol{\sigma}\|_{2}^{2}}{m_{b} K^{2}}\right) .
\end{aligned}
$$

Under the Cauchy-Schwarz's inequality, i.e., $\boldsymbol{b}_{1}{ }^{T} \boldsymbol{b}_{2} \leq$ $\left\|\boldsymbol{b}_{1}\right\|\left\|\boldsymbol{b}_{2}\right\| \leq \frac{\left\|\boldsymbol{b}_{1}\right\|^{2}}{2}+\frac{\left\|\boldsymbol{b}_{2}\right\|^{2}}{2}$, we further have

$$
\begin{aligned}
& \mathbb{E}\left[F\left(\mathbf{w}^{(n+1)}\right)-F\left(\mathbf{w}^{(n)}\right)\right] \\
& \leq-\eta\left\|\nabla F\left(\mathbf{w}^{(n)}\right)\right\|^{2}+\frac{\eta^{2}\left\|\nabla F\left(\mathbf{w}^{(n)}\right)\right\|^{2}}{2}+\frac{\left\|\mathbb{E}\left[\varepsilon^{(n)}\right]\right\|^{2}}{2} \\
& +\frac{L \eta^{2}}{2} \mathbb{E}\left[\left\|\varepsilon^{(n)}\right\|^{2}\right]+\frac{\eta^{2} L^{2}\left\|\mathbb{E}\left[\varepsilon^{(n)}\right]\right\|^{2}}{2} \\
& +\frac{L \eta^{2}}{2}\left(\left\|\nabla F\left(\mathbf{w}^{(n)}\right)\right\|_{2}^{2}+\frac{\|\boldsymbol{\sigma}\|_{2}^{2}}{m_{b} K^{2}}\right)+\frac{\eta^{2}\left\|\nabla F\left(\mathbf{w}^{(n)}\right)\right\|^{2}}{2} \\
& =-\eta\left(1-\eta-\frac{\eta L}{2}\right)\left\|\nabla F\left(\mathbf{w}^{(n)}\right)\right\|^{2}+\frac{\eta^{2} L\|\boldsymbol{\sigma}\|_{2}^{2}}{2 m_{b} K^{2}} \\
& +\frac{\left(1+\eta^{2} L^{2}\right)\left\|\mathbb{E}\left[\varepsilon^{(n)}\right]\right\|^{2}}{2}+\frac{\eta^{2} L}{2} \mathbb{E}\left[\left\|\varepsilon^{(n)}\right\|^{2}\right] \\
& \leq-\frac{\eta}{2}\left\|\nabla F\left(\mathbf{w}^{(n)}\right)\right\|^{2}+\frac{\left(1+\eta^{2} L^{2}\right)\left\|\mathbb{E}\left[\varepsilon^{(n)}\right]\right\|^{2}}{2} \\
& +\frac{\eta^{2} L\|\boldsymbol{\sigma}\|_{2}^{2}}{2 m_{b} K^{2}}+\frac{\eta^{2} L}{2} \mathbb{E}\left[\left\|\varepsilon^{(n)}\right\|^{2}\right],
\end{aligned}
$$

where the inequality in (43) follows that $\eta \leq \frac{2}{2+L}$. Next, by applying Assumption 2, it hence follows that

$$
\begin{aligned}
& \mathbb{E}\left[F\left(\mathbf{w}^{(n+1)}\right)\right]-F^{\star} \leq(1-\delta \eta)\left(\mathbb{E}\left[F\left(\mathbf{w}^{(n)}\right)\right]-F^{\star}\right) \\
& +\frac{\left(1+\eta^{2} L^{2}\right)\left\|\mathbb{E}\left[\varepsilon^{(n)}\right]\right\|^{2}}{2}+\frac{\eta^{2} L\|\boldsymbol{\sigma}\|_{2}^{2}}{2 m_{b} K^{2}}+\frac{\eta^{2} L}{2} \mathbb{E}\left[\left\|\varepsilon^{(n)}\right\|^{2}\right] .
\end{aligned}
$$

Through some further algebraic manipulation over the above inequality based on $m_{b}=N$ and $C=1-\delta \eta$, we have (17). This thus completes the proof.

\section{B. Proof of Proposition 3}

Notice that problem (P1.1) is convex, and thus strong duality holds between problem (P1.1) and its Lagrange dual problem [50]. Hence, we leverage the Lagrange duality method to optimally solve problem (P1.1). Let $\varphi_{k} \geq 0, \forall k \in \mathcal{K}$ denote the dual variable associated with the $k$-th constraint in (29), respectively. The partial Lagrangian of problem (P1.1) is thus given by

$$
\begin{aligned}
\mathcal{L}_{1}\left(\left\{\hat{p}_{k}^{(n)}\right\}\right)= & \sum_{n=1}^{N} J^{(n)} A^{(n)}\left(\sum_{k \in \mathcal{K}} h_{k}^{(n)} \hat{p}_{k}^{(n)}-K\right)^{2} \\
& +\sum_{n=1}^{N} J^{(n)} B^{(n)} \sum_{k \in \mathcal{K}}\left(h_{k}^{(n)} \hat{p}_{k}^{(n)}-1\right)^{2} \\
& +\sum_{k \in \mathcal{K}} \varphi_{k}\left(\frac{1}{N} \sum_{n \in \mathcal{N}}\left(\hat{p}_{k}^{(n)}\right)^{2} \hat{G}^{(n)}-P_{k}^{\text {ave }}\right) .
\end{aligned}
$$

Then the dual function is

$$
W_{1}\left(\left\{\varphi_{k}\right\}\right)=\min _{\left\{0 \leq q_{k}^{(n)} \leq P_{k, n}^{\max }\right\}} \mathcal{L}_{1}\left(\left\{\hat{p}_{k}^{(n)}\right\}\right) .
$$

The dual problem of problem (P1) is given as

$$
\text { D1 : } \min _{\left\{\varphi_{k} \geq 0\right\}} W_{2}\left(\left\{\varphi_{k}\right\}\right) \text {. }
$$

Due to the strong duality between problems (P1.1) and (D1), we solve problem (P1.1) by equivalently solving its dual problem (D1). Let $\left\{\hat{p}_{k}^{(n) \text { opt }}\right\}$ denote the optimal primal solution to problem (P1.1), and $\left\{\varphi_{k}^{\mathrm{opt}}\right\}$ the optimal dual solution to 
problem (D1). We first evaluate the dual function $W_{1}\left(\left\{\varphi_{k}\right\}\right)$ under any given feasible $\left\{\varphi_{k}\right\}$, and then obtain the optimal dual variables $\left\{\varphi_{k}^{\mathrm{opt}}\right\}$ to maximize $\left.W_{1}\left(\left\{\varphi_{k}\right\}\right\}\right)$.

First, we obtain $\left.W_{1}\left(\left\{\varphi_{k}\right\}\right\}\right)$ by solving problem (44) under any given feasible $\left\{\varphi_{k}\right\}$, which can be decomposed into a series of subproblems each for one communication round $n$ as follows.

$$
\begin{aligned}
& \min _{\left\{q_{k}^{(n)}\right\}} J^{(n)} A^{(n)}\left(\sum_{k \in \mathcal{K}} h_{k}^{(n)} \hat{p}_{k}^{(n)}-K\right)^{2}+\frac{\varphi_{k} \hat{G}^{(n)}\left(\hat{p}_{k}^{(n)}\right)^{2}}{N} \\
&+J^{(n)} B^{(n)} \sum_{k \in \mathcal{K}}\left(h_{k}^{(n)} \hat{p}_{k}^{(n)}-1\right)^{2} \\
& \text { s.t. } 0 \leq q_{k}^{(n)} \leq P_{k, n}^{\max }, \forall k \in \mathcal{K} .
\end{aligned}
$$

By taking its first-order derivation w.r.t. each $\hat{p}_{k}^{(n)}$, we have the following lemma.

Lemma 1. The optimal solution to problem (46) denoted by $q_{k}^{(n) \star}, \forall k \in \mathcal{K}, n \in \mathcal{N}$ is given as

$$
\hat{p}_{k}^{(n) \star}=\min \left[\frac{B^{(n)}+A^{(n)} K}{M_{k}^{(n)}+A^{(n)} M_{k}^{(n)} \sum_{i \in \mathcal{K}} \frac{h_{i}^{(n)}}{M_{i}^{(n)}}}, P_{k, n}^{\max }\right],
$$

where $M_{k}^{(n)} \triangleq B^{(n)} h_{k}^{(n)}+\frac{\varphi_{k} \hat{G}^{(n)}}{N J^{(n)} h_{k}^{(n)}}, \forall k \in \mathcal{K}, n \in \mathcal{N}$.

With Lemma 1 problem (44) is solved under any given $\left\{\varphi_{k}\right\}$, and the dual function $\left.W_{1}\left(\left\{\varphi_{k}\right\}\right\}\right)$ is accordingly obtained. It remains to find the optimal $\left\{\varphi_{k} \geq 0\right\}$. Since the dual function $W_{1}\left(\left\{\varphi_{k}\right\}\right)$ is concave but non-differentiable in general, one can use subgradient based methods such as the ellipsoid method [52] to obtain the optimal dual variables. Note that for the objective function in (44), the subgradient w.r.t. $\varphi_{k}, \forall k$, is $\frac{1}{N} \sum_{n \in \mathcal{N}}\left(\hat{p}_{k}^{(n) \star}\right)^{2} \hat{G}^{(n)}-P_{k}^{\text {ave }}$. By replacing $\left\{\varphi_{k}\right\}$ in Lemma 1 with the obtained optimal dual variables $\left\{\varphi_{k}^{\text {opt }}\right\}$, the optimal solution to problem (P1.1) is accordingly obtained as shown in Proposition 3 . This thus completes the proof.

\section{Proof of Proposition 4}

Notice that problem (P2.1) is convex and satisfies the Slater's condition, and thus strong duality holds between problem (P2.1) and its Lagrange dual problem [50]. Therefore, we apply the Lagrange duality method to optimally solve problem (P2.1). Let $\mu_{n}$ and $\lambda_{k} \geq 0$ denote the dual variable associated with the $n$-th constraints in (35) and the $k$-th constraint in (37), respectively. Then the partial Lagrangian of problem $(\mathrm{P} 2.1)$ is

$$
\begin{aligned}
\mathcal{L}\left(\left\{q_{k}^{(n)}\right\}\right)= & \sum_{n=1}^{N} J^{(n)} B^{(n)} \sum_{k \in \mathcal{K}}\left(h_{k}^{(n)} q_{k}^{(n)}-1\right)^{2} \\
& +\sum_{k \in \mathcal{K}} \lambda_{k}\left(\frac{1}{N} \sum_{n \in \mathcal{N}}\left(q_{k}^{(n)}\right)^{2} \hat{G}^{(n)}-P_{k}^{\text {ave }}\right) \\
& +\sum_{n \in \mathcal{N}} \mu_{n}\left(\sum_{k \in \mathcal{K}} h_{k}^{(n)} q_{k}^{(n)}-K\right)
\end{aligned}
$$

Then the dual function is

$$
\begin{gathered}
W_{2}\left(\left\{\lambda_{k}\right\},\left\{\mu_{n}\right\}\right)=\min _{\left\{q_{k}^{(n)} \geq 0\right\}} \mathcal{L}\left(\left\{q_{k}^{(n)}\right\}\right) \\
\text { s.t. (36). }
\end{gathered}
$$

Accordingly, the dual problem of problem (P1) is given as

$$
\text { D2 : } \min _{\left\{\lambda_{k} \geq 0\right\},\left\{\mu_{n}\right\}} W_{2}\left(\left\{\lambda_{k}\right\},\left\{\mu_{n}\right\}\right) \text {. }
$$

Due to the fact that the strong duality holds between problems (P2.1) and (D2), we can solve problem (P2.1) by equivalently solving its dual problem (D2). For notational convenience, let $\left\{q_{k}^{(n) \text { opt }}\right\}$ denote the optimal primal solution to problem (P2.1), and $\left\{\lambda_{k}^{\text {opt }}\right\}$ and $\left\{\mu_{n}^{\text {opt }}\right\}$ denote the optimal dual solution to problem (D2). In the following, we first evaluate the dual function $W_{2}\left(\left\{\lambda_{k}\right\},\left\{\mu_{n}\right\}\right)$ under any given feasible $\left\{\lambda_{k}\right\}$ and $\left\{\mu_{n}\right\}$, and then obtain the optimal dual variables $\left\{\lambda_{k}^{\mathrm{opt}}\right\}$ and $\left\{\mu_{n}^{\text {opt }}\right\}$ to maximize $W_{2}\left(\left\{\lambda_{k}\right\},\left\{\mu_{n}\right\}\right)$.

First, we obtain $W_{2}\left(\left\{\lambda_{k}\right\},\left\{\mu_{n}\right\}\right)$ by solving problem (49) under any given feasible $\left\{\lambda_{k}\right\}$ and $\left\{\mu_{n}\right\}$, which can be decomposed into a sequence of subproblems each for optimizing the power scaling factor in edge device $k$ at one communication round $n$, i.e.,

$\min _{q_{k}^{(n)}} J^{(n)} B^{(n)}\left(h_{k}^{(n)} q_{k}^{(n)}-1\right)^{2}+\frac{\lambda_{k} \hat{G}^{(n)}}{N}\left(q_{k}^{(n)}\right)^{2}+\mu_{n} h_{k}^{(n)} q_{k}^{(n)}$

s.t. $0 \leq q_{k}^{(n)} \leq P_{k, n}^{\max }$

Via the first-order derivation of the objective function in (51), we have the following lemma.

Lemma 2. The optimal solution to problem (51) denoted by $q_{k}^{(n) \star}, \forall k \in \mathcal{K}, n \in \mathcal{N}$ is given as

$$
q_{k}^{(n) \star}=\min \left[\frac{\left(h_{k}^{(n)}-\frac{\mu_{n} h_{k}^{(n)}}{2 J^{(n)} B^{(n)}}\right)^{+}}{\left(h_{k}^{(n)}\right)^{2}+\frac{2 \lambda_{k} \hat{G}^{(n)}}{N J^{(n)} B^{(n)}}}, P_{k, n}^{\max }\right] .
$$

Therefore, with Lemma 2 problem (49) is solved, and the dual function $W_{2}\left(\left\{\lambda_{k}\right\},\left\{\mu_{n}\right\}\right)$ is accordingly obtained. It remains to find the optimal $\left\{\lambda_{k} \geq 0\right\}$ and $\left\{\mu_{n}\right\}$. Since the dual function $W_{2}\left(\left\{\lambda_{k}\right\},\left\{\mu_{n}\right\}\right)$ is concave but nondifferentiable in general, we can use the ellipsoid method [52], to obtain the optimal dual variables. Note that for the objective function in (49), the subgradient w.r.t. $\lambda_{k}, \forall k$, is $\frac{1}{N} \sum_{n \in \mathcal{N}}\left(q_{k}^{(n) \star}\right)^{2} \hat{G}^{(n)}-P_{k}^{\text {ave }}$, while that for dual variables $\mu_{n}, \forall n \in \mathcal{N}$, is $\sum_{k \in \mathcal{K}} h_{k}^{(n)} q_{k}^{(n) \star}-K$. By replacing $\left\{\lambda_{k}\right\}$ and $\left\{\mu_{n}\right\}$ in Lemma 2 with the obtained optimal dual variables $\left\{\lambda_{k}^{\text {opt }}\right\}$ and $\left\{\mu_{n}^{\text {opt }}\right\}$, the optimal solution to problem (P2.1) is accordingly obtained as shown in Lemma 4. This thus completes the proof.

\section{REFERENCES}

[1] X. Cao, G. Zhu, J. Xu, and S. Cui, "Optimized power control for overthe-air federated edge learning," in Proc. IEEE ICC, Jun. 2021, pp. 1-6.

[2] K. B. Letaief, W. Chen, Y. Shi, J. Zhang, and Y.-J. A. Zhang, "The roadmap to 6G: AI empowered wireless networks," IEEE Commun. Mag., vol. 57, no. 8, pp. 84-90, Aug. 2019.

[3] G. Zhu, D. Liu, Y. Du, C. You, J. Zhang, and K. Huang, "Toward an intelligent edge: Wireless communication meets machine learning," IEEE Commun. Mag., vol. 58, no. 1, pp. 19-25, Jan. 2020. 
[4] J. Park, S. Samarakoon, M. Bennis, and M. Debbah, "Wireless network intelligence at the edge," Proc. IEEE, vol. 107, no. 11, pp. 2204-2239, Nov. 2019.

[5] N. H. Tran, W. Bao, A. Zomaya, M. N. H. Nguyen, and C. S. Hong, "Federated learning over wireless networks: Optimization model design and analysis," in Proc. IEEE INFOCOM, Apr. 2019, pp. 1387-1395.

[6] J. Konečný, H. B. McMahan, F. X. Yu, P. Richtárik, A. T. Suresh, and D. Bacon, "Federated learning: Strategies for improving communication efficiency," 2016. [Online]. Available: https://arxiv.org/pdf/1610.05492.pdf

[7] M. Chen, Z. Yang, W. Saad, C. Yin, H. V. Poor, and S. Cui, "A joint learning and communications framework for federated learning over wireless networks," IEEE Trans. Wireless Commun., vol. 20, no. 1, pp. 269-283, Jan. 2021

[8] J. Park, S. Samarakoon, A. Elgabli, J. Kim, M. Bennis, S.-L. Kim, and M. Debbah, "Communication-efficient and distributed learning over wireless networks: Principles and applications," Proc. IEEE, vol. 109, no. 5, pp. 796-819, May 2021.

[9] X. Mo and J. Xu, "Energy-efficient federated edge learning with joint communication and computation design," J. Commun. Inf. Net., vol. 6 , no. 2, pp. 110-124, Jun. 2021.

[10] J. Ren, Y. He, D. Wen, G. Yu, K. Huang, and D. Guo, "Scheduling for cellular federated edge learning with importance and channel awareness," IEEE Trans. Wireless Commun., vol. 19, no. 11, pp. 7690-7703, Nov. 2020.

[11] G. Zhu, J. Xu, K. Huang, and S. Cui, "Over-the-air computing for wireless data aggregation in massive IoT," IEEE Wireless Commun., vol. 28, no. 4, pp. 57-65, Aug. 2021.

[12] B. Nazer and M. Gastpar, "Computation over multiple-access channels," IEEE Trans. Inf. Theory, vol. 53, no. 10, pp. 3498-3516, Oct. 2007.

[13] M. Gastpar, "Uncoded transmission is exactly optimal for a simple Gaussian sensor network," IEEE Trans. Inf. Theory, vol. 54, no. 11, pp. 5247-5251, Nov. 2008.

[14] O. Abari, H. Rahul, D. Katabi, and M. Pant, "Airshare: Distributed coherent transmission made seamless," in Proc. IEEE INFOCOM, Kowloon, Hong Kong, Apr. 2015, pp. 1742-1750.

[15] X. Cao, G. Zhu, J. Xu, and K. Huang, "Optimized power control for over-the-air computation in fading channels," IEEE Trans. Wireless Commun., vol. 19, no. 11, pp. 7498-7513, Nov. 2020.

[16] W. Liu, X. Zang, Y. Li, and B. Vucetic, "Over-the-air computation systems: Optimization, analysis and scaling laws," IEEE Trans. Wireless Commun., vol. 19, no. 8, pp. 5488-5502, Aug. 2020.

[17] X. Cao, G. Zhu, J. Xu, and K. Huang, "Cooperative interference management for over-the-air computation networks," IEEE Trans. Wireless Commun., vol. 20, no. 4, pp. 2634-2651, Apr. 2021.

[18] G. Zhu and K. Huang, "MIMO over-the-air computation for highmobility multimodal sensing," IEEE Internet Things J., vol. 6, no. 4, pp. 6089-6103, Aug. 2019.

[19] L. Chen, N. Zhao, Y. Chen, F. R. Yu, and G. Wei, "Over-the-air computation for IoT networks: Computing multiple functions with antenna arrays," IEEE Internet Things J., vol. 5, no. 6, pp. 5296-5306, Dec. 2018.

[20] X. Zhai, X. Chen, J. Xu, and D. W. K. Ng, "Hybrid beamforming for massive MIMO over-the-air computation," IEEE Trans. Commun., vol. 69, no. 4, pp. 2737-2751, Apr. 2021.

[21] J. Dong, Y. Shi, and Z. Ding, "Blind over-the-air computation and data fusion via provable Wirtinger flow," IEEE Trans. Signal Process., vol. 68, pp. 1136-1151, Jan. 2020.

[22] T. Sery and K. Cohen, "On analog gradient descent learning over multiple access fading channels," IEEE Trans. Signal Process., vol. 68, pp. 2897-2911, 2020.

[23] G. Zhu, Y. Wang, and K. Huang, "Broadband analog aggregation for low-latency federated edge learning," IEEE Trans. Wireless Commun., vol. 19, no. 1, pp. 491-506, Jan. 2020.

[24] Y. Sun, S. Zhou, Z. Niu, and D. Gündüz, "Dynamic scheduling for over-the-air federated edge learning with energy constraints," 2021. [Online]. Available: https://arxiv.org/pdf/2106.00490.pdf

[25] S. Xia, J. Zhu, Y. Yang, Y. Zhou, Y. Shi, and W. Chen, "Fast convergence algorithm for analog federated learning," in Proc. IEEE ICC, Jun. 2021, pp. 1-6.

[26] X. Fan, Y. Wang, Y. Huo, and Z. Tian, "Joint optimization of communications and federated learning over the air," 2021. [Online]. Available: https://arxiv.org/pdf/2104.03490.pdf

[27] K. Yang, T. Jiang, Y. Shi, and Z. Ding, "Federated learning via overthe-air computation," IEEE Trans. Wireless Commun., vol. 19, no. 3, pp. 2022-2035, Mar. 2020.
[28] S. Wang, Y. Hong, R. Wang, Q. Hao, Y.-C. Wu, and D. W. K. Ng, "Edge federated learning via unit-modulus over-the-air computation," 2021. [Online]. Available: https://arxiv.org/pdf/2101.12051.pdf

[29] M. Mohammadi Amiri and D. Gündüz, "Machine learning at the wireless edge: Distributed stochastic gradient descent over-the-air," IEEE Trans. Signal Process., vol. 68, pp. 2155-2169, 2020.

[30] M. M. Amiri and D. Gündüz, "Federated learning over wireless fading channels," IEEE Trans. Wireless Commun., vol. 19, no. 5, pp. 35463557, May 2020

[31] X. Fan, Y. Wang, Y. Huo, and Z. Tian, "1-bit compressive sensing for efficient federated learning over the air," 2021. [Online]. Available: https://arxiv.org/pdf/2103.16055.pdf

[32] H. Guo, A. Liu, and V. K. N. Lau, "Analog gradient aggregation for federated learning over wireless networks: Customized design and convergence analysis," IEEE Internet Things J., vol. 8, no. 1, pp. 197210, Jan. 2021

[33] C. Xu, S. Liu, Z. Yang, Y. Huang, and K.-K. Wong, "Learning rate optimization for federated learning exploiting over-the-air computation," IEEE J. Sel. Areas Commun., pp. 1-1, 2021.

[34] J. Zhang, N. Li, and M. Dedeoglu, "Federated learning over wireless networks: A band-limited coordinated descent approach," 2021. [Online]. Available: https://arxiv.org/pdf/2102.07972.pdf

[35] G. Zhu, Y. Du, D. Gündüz, and K. Huang, "One-bit over-the-air aggregation for communication-efficient federated edge learning: Design and convergence analysis," IEEE Trans. Wireless Commun., vol. 20, no. 3, pp. 2120-2135, Mar. 2021.

[36] R. Jiang and S. Zhou, "Cluster-based cooperative digital over-the-air aggregation for wireless federated edge learning," in Proc. IEEE/CIC ICCC, Aug. 2020, pp. 887-892.

[37] D. Liu and O. Simeone, "Privacy for free: Wireless federated learning via uncoded transmission with adaptive power control," IEEE J. Sel. Areas Commun., vol. 39, no. 1, pp. 170-185, Jan. 2021.

[38] Y. Koda, K. Yamamoto, T. Nishio, and M. Morikura, "Differentially private AirComp federated learning with power adaptation harnessing receiver noise," in Proc. IEEE GLOBECOM, Dec. 2020, pp. 1-6.

[39] A. Sonee and S. Rini, "Efficient federated learning over multiple access channel with differential privacy constraints," 2020. [Online]. Available: https://arxiv.org/pdf/2005.07776.pdf

[40] M. Seif, R. Tandon, and M. Li, "Wireless federated learning with local differential privacy," in Proc. IEEE ISIT, Jun. 2020, pp. 2604-2609.

[41] C. Shen, J. Xu, S. Zheng, and X. Chen, "Resource rationing for wireless federated learning: Concept, benefits, and challenges," 2021. [Online]. Available: https://arxiv.org/pdf/2104.06990.pdf

[42] N. Zhang and M. Tao, "Gradient statistics aware power control for overthe-air federated learning," IEEE Trans. Wireless Commun., vol. 20 no. 8, pp. 5115-5128, Aug. 2021.

[43] C. Hou, K. K. Thekumparampil, G. Fanti, and S. Oh, "Reducing the communication cost of federated learning through multistage optimization," 2021. [Online]. Available: https://arxiv.org/pdf/2108.06869.pdf

[44] B. McMahan, E. Moore, D. Ramage, S. Hampson, and B. Arcas, "Communication-efficient learning of deep networks from decentralized data," in Proc. International Conference on Artificial Intelligence and Statistics, vol. 54, Fort Lauderdale, FL, USA, 20-22 Apr. 2017, pp. 1273-1282.

[45] M. P. Friedlander and M. Schmidt, "Hybrid deterministic-stochastic methods for data fitting," SIAM J. Sci. Comput., vol. 3, no. 34, pp. 1380-1405, May 2012.

[46] X. Li, K. Huang, W. Yang, S. Wang, and Z. Zhang, "On the convergence of FedAvg on non-IID data," 2019. [Online]. Available: https://arxiv.org/pdf/1907.02189.pdf

[47] H. Karimi, J. Nutini, and M. Schmidt, "Linear convergence of gradient and proximal-gradient methods under the Polyak-Łojasiewicz condition," Lecture Notes in Computer Science, pp. 795-811, 2016. [Online]. Available: http://dx.doi.org/10.1007/978-3-319-46128-1_50

[48] J. Bernstein, Y.-X. Wang, K. Azizzadenesheli, and A. Anandkumar, "signSGD: Compressed optimisation for non-convex problems," in Proc. International Conference on Machine Learning (ICML), vol. 80, 10-15 Jul. 2018, pp. 560-569.

[49] L. Bottou, F. E. Curtis, and J. Nocedal, "Optimization methods for largescale machine learning," SIAM Review, vol. 60, no. 2, pp. 223-311, 2018.

[50] M. Grant and S. Boyd, "CVX: Matlab software for disciplined convex programming," 2016. [Online]. Available: http://cvxr.com/cvx

[51] F. Ang, L. Chen, N. Zhao, Y. Chen, W. Wang, and F. R. Yu, "Robust federated learning with noisy communication," IEEE Trans. Commun., vol. 68, no. 6, pp. 3452-3464, Jun. 2020. 
[52] S. Boyd, "Ellipsoid method." [Online]. Available: https://web.stanford.edu/class/ee364b/lectures/ellipsoidmethodslides.pdf 